\title{
Review \\ Recent Advancements in Plant-Derived Nanomaterials Research for Biomedical Applications
}

\author{
Rashmi Trivedi ${ }^{1}$, Tarun Kumar Upadhyay ${ }^{1, * \mathbb{D}}$, Mohd Hasan Mujahid ${ }^{1}$, Fahad Khan ${ }^{2} \mathbb{D}$, Pratibha Pandey ${ }^{2} \mathbb{D}$, \\ Amit Baran Sharangi ${ }^{3}{ }^{(D}$, Khursheed Muzammil ${ }^{4}\left(\mathbb{D}\right.$, Nazim Nasir $^{5}{ }^{\mathbb{D}}$, Atiq Hassan ${ }^{5}$, Nadiyah M. Alabdallah ${ }^{6}$, \\ Sadaf Anwar ${ }^{7}$, Samra Siddiqui ${ }^{8}$ and Mohd Saeed ${ }^{9}$ (D)
}

\section{check for}

updates

Citation: Trivedi, R.; Upadhyay, T.K.; Mujahid, M.H.; Khan, F.; Pandey, P.; Sharangi, A.B.; Muzammil, K.; Nasir, N.; Hassan, A.; Alabdallah, N.M.; et al. Recent Advancements in Plant-Derived Nanomaterials Research for Biomedical Applications. Processes 2022, 10, 338. https:// doi.org/10.3390/pr10020338

Academic Editor: Diego

Gamba-Sánchez

Received: 5 January 2022

Accepted: 2 February 2022

Published: 10 February 2022

Publisher's Note: MDPI stays neutral with regard to jurisdictional claims in published maps and institutional affiliations.

Copyright: (C) 2022 by the authors. Licensee MDPI, Basel, Switzerland. This article is an open access article distributed under the terms and conditions of the Creative Commons Attribution (CC BY) license (https:// creativecommons.org/licenses/by/ $4.0 /)$
1 Department of Biotechnology, Parul Institute of Applied Sciences and Animal Cell Culture AndImmunobio Chemistry Lab, Centre of Research for Development, Parul University, Vadodara 391760, India; rashmitrivedi888@gmail.com (R.T.); mhasanmujahid@gmail.com (M.H.M.)

2 Department of Biotechnology, Noida Institute of Engineering \& Technology, Greater Noida 201306, India; fahadintegralian@gmail.com (F.K.); shukla.pratibha1985@gmail.com (P.P.)

3 Department of Plantation, Spices, Medicinal \& Aromatic Crops, BCKV-Agricultural University, Mohanpur 741252, India; absharangi@gmail.com

4 Department of Public Health, Khamis Mushayt Campus, College of Applied Medical Sciences, King Khalid University, Abha 62559, Saudi Arabia; ktahir@kku.edu.sa

5 Department of Basic Medical Sciences, Khamis Mushait Campus, College of Applied Medical Sciences, King Khalid University, Abha 62559, Saudi Arabia; drnnasir@gmail.com (N.N.); atiqhassan@gmail.com (A.H.)

6 Department of Biology, College of Science, Imam Abdulrahman Bin Faisal University, Dammam 31441, Saudi Arabia; nmalabdallah@iau.edu.sa

7 Department of Biochemistry, College of Medicine, University of Hail, Hail 34464, Saudi Arabia; sadaf18anwar@gmail.com

8 College of Preparatory, University of Hail, Hail 34464, Saudi Arabia; s.siddiqui@uoh.edu.sa

9 Department of Biology, College of Sciences, University of Hail, Hail 34464, Saudi Arabia; mo.saeed@uoh.edu.sa

* Correspondence: tarun_bioinfo@yahoo.co.in

\begin{abstract}
Engineering, physics, chemistry, and biology are all involved in nanotechnology, which comprises a wide variety of multidisciplinary scientific field devices. The holistic utilization of metallic nanoparticles in the disciplines of bio-engineering and bio-medicine has attracted a great deal of attention. Medical nanotechnology research can offer immense health benefits for humans. While the advantages of developing nanomaterials have been well documented, it is precisely apparent that there are still some major issues that remain unattended to those need to be resolved immediately so as to ensure that they do not adversely affect living organisms in any manner. The existence of nanoparticles gives them particular value in biology and materials science, as an emerging scientific field, with multiple applications in science and technology, especially with numerous frontiers in the development of new materials. Presented here is a review of recent noteworthy developments regarding plant-derived nanomaterials and their use in the development of medicine and biomedical applications around the world.
\end{abstract}

Keywords: nanomaterials; nanoparticles; plants; biomedical; drug delivery

\section{Introduction to Nanoparticles}

Nanomaterials have brought a revolution in the area of biomedical research due to having a high loading capacity and strong protection to the payloads. Nanoparticles (NP) have a variety of choices in biomedicine, bio-labeling, agriculture, antimicrobial agents, and in many other areas [1]. As a result of their wide range of applications in a variety of fields, nanoparticles have become a hot topic of study in recent years, including in diagnostics, biomarkers, cell identification, antimicrobials, drug delivery, and cancer therapies [2]. Physical, chemical, and biological methods can all be used to synthesize and develop nanoparticles. However, biological methods are regarded to be an especially appealing 
method as they are simple, cheap, and can be modified to achieve the desired shape, size, and functions [3,4]. NPs synthesized by the top-down method utilize physical techniques, such as grinding, diffusion, thermal decomposition, irradiation, etc., to break the bulky material into small particles. According to the bottom-up method of nano-particle synthesis, chemical and biological processes are used for NPs synthesis [5]. While the above synthetic processes use chemical agents that are environmentally damaging, toxic by-products can also be formed due to the use of environmentally corrosive chemicals. The use of NPs has attracted increasing interest from researchers for biomedical research due to their highly specific cell and tissue interacting properties and high efficiency fighting diseases [6]. Moreover, plant-derived nano-particles have developed as new therapeutic approaches to treat a variety of diseases. This emerging green nanotechnology can now be used to generalize the synthesis of metallic nanoparticles by plant-mediated green synthesis, which presents a remarkable breakthrough in the green synthesis of metal nanoparticles. Some of the metal-based nanoparticles have been found to show antimicrobial properties, and sometimes these may work as novel materials to stop the spread of resistance to antimicrobial agents [7]. Further development for the synthesis of nanoparticles takes place mainly concerning their chemical composition, size and shape of the substance. The increased particle size and the surface area-to-volume ratio entering the region where quantum effects prevail are two of the main contributing factors to the physiochemical properties of nanoparticles [8]. Nanomaterials actually have absolute properties in comparison with bulk materials, as their surface area-to-volume ratio is extremely high, and exponentially increases as particle size decreases. In the future, nanotechnology may be used to solve many central questions about biological systems that are currently a burden on society. NPs have a few of their dimensions at nanoscale making their properties different from the particles at the molecular or atomic level, and there is a requirement to take full advantage of the nanoscale payload delivery and transport properties of NPs [9]. The physical properties of nanoparticles have changed as a function of their size, including melting point, electrical conductivity, fluorescence, chemical reactivity, and magnetic permeability [10]. These exemplary features make nanomaterials tunable and promising for the study of biological phenomena. The sizes of functional elements present in a biological system such as DNA, RNA, and proteins are nanometric, promoting easier interactions of NPs with these functional molecules [11]. Over the past decade, nanotechnology has gained significant attention across an extensive range of applications, including in medicine, energy, healthcare, the environment, cosmetics, and drugs. For utilization in the medical field, the NPs should be of definite size because sizably voluminous NPs will be phagocytosed and expeditiously eliminated from the circulatory system, while profoundly diminutive NPs can become toxic and will undergo an expeditious abstraction from the kidney [1-14]. Nanomedicine can be applied as the application of various nanotechnology-based tools for the development of efficient solutions to overcome medical challenges and the management of several diseases. As a development in nanotechnology, it should be appreciated that the field does not represent an isolated disciplinary field, but rather the meeting of sundry traditionally oriented sciences, including biology, chemistry, physics, and materials science, in order to synthesize the collaborative expertise needed to advance these incipient technologies [15]. A new field of research has been created by the application of nanotechnology to medical research, offering scientists and researchers an entirely new way of studying biological systems. By manipulating materials at the nanometer scale, it is possible to change their fundamental physical characteristics and bioactivity; these tools can provide control over measures such as altered solubility and retention time of the blood pool, controlled release for short or long periods, environmental triggers, or the highly targeted delivery of drugs [16]. Targeted drug delivery would enable the drug to remain stable, absorb, and concentrate more strongly throughout the target tissue and also permit its release at the target site to be reproducible and long-lasting [17,18]. This review shows an overview of nanotechnology's potential in targeted drug delivery, the development of 
medicine, and diagnostics. Various anticancerous and antimicrobial properties of NPs are shown in Figure 1.
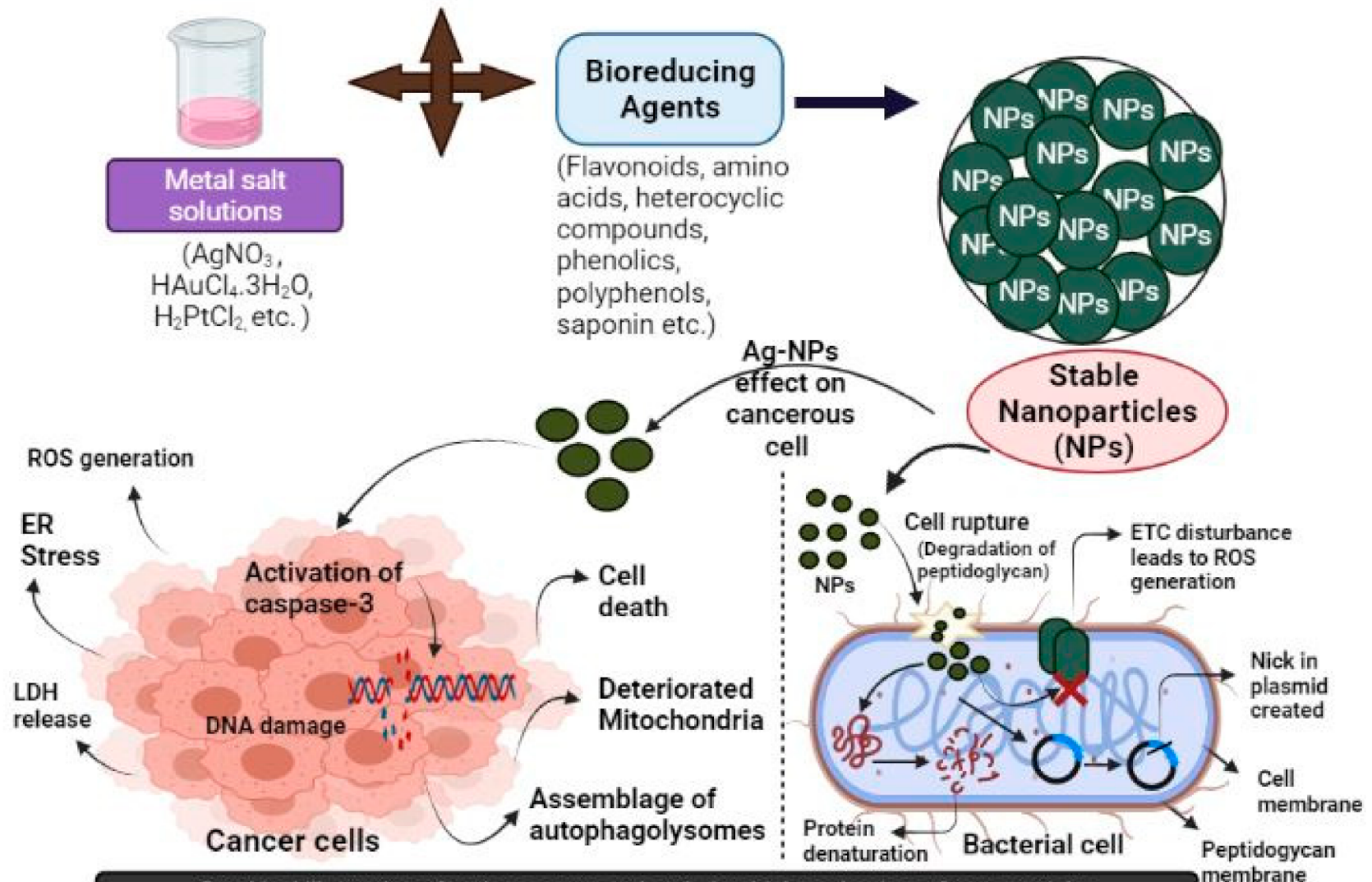

Graphical illustration of anticancerous and antimicrobial mechanism of nanoparticles

Figure 1. Metal salt solutions $\left(\mathrm{AgNO}_{3}, \mathrm{HAuCl}_{4} \cdot 3 \mathrm{H} 2 \mathrm{O}, \mathrm{H}_{2} \mathrm{PtCl}_{2}\right)$ after treatment with bioreducing agents (flavonoids, amino acids, heterocyclic compounds, phenolics, polyphenols, saponins, etc.) form stable nanoparticles. These nanoparticles (Ag-NPs), when treated on cancerous cells, activates the Caspase-3 pathway, which subsequently activates the different cellular process such as ROS generation, accumulation of autophagolysomes, ER stress generated, LDH releases, and mitochondria disrupted, and eventually leads to cell collapse, hence cell death. The NPs degrade the peptidoglycan layer of bacterial cell wall. These NPs disturb the electron transport chain (ETC), which eventually leads to reactive oxygen species (ROS) generation. They intercalate with the protein and leading to its denaturation. Furthermore, NPs also create nicks in the plasmid. All these events inside the bacterial cell lead to its death.

\section{Different Types of Nanoparticles}

Type of nanoparticles varies with the process by which nanoparticles are synthesized, as shown in the Figure 2. Nanoparticles synthesised from plants presents a preferable source of NP synthesis. The synthesis of NPs from plants has many advantages over physical and chemical modes of synthesis. It is more straightforward, rapid, and cost effective than chemical or physical nanoparticles synthesis and uses less energy and takes place under moderate operating conditions. Almost all parts of the plant, including the root, stem, latex, leaf, and bark, can be used for the synthesis of NPs. Although the exact mechanism to synthesize NPs from plants is ambiguous, still, it has been found that phenols, flavanoids and many more biomolecules present in plants can help in the reduction of metal ions resulting in the synthesis of NPs. Due to their abundance of capabilities and bioactive reducing metabolites in many forms, the biological synthesis of nanomaterials, in particular silver nanoparticles, has received a significant amount of attention. 


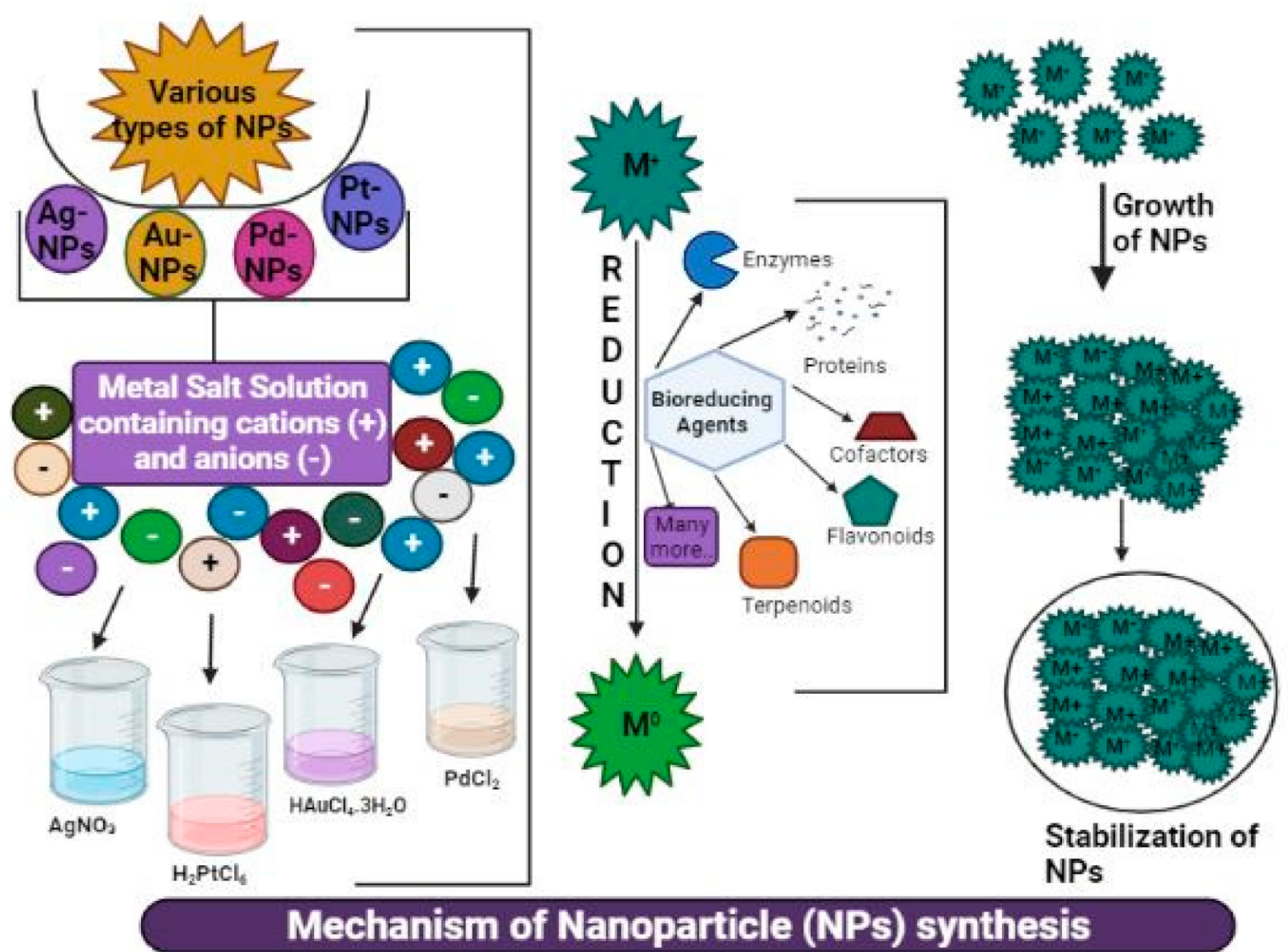

Figure 2. The synthesis of nanoparticles is shown through bio-reduction method. Metal is being reduced by metal salt solution $\left(\mathrm{M}^{+} \rightarrow \mathrm{M}^{0}\right)$ to yield NPs with the help of various bio-reducing agents (enzymes, proteins, cofactors, flavonoids, terpenoids, etc.).

\subsection{Metallic Nanoparticles}

\subsubsection{Silver Nanoparticles}

Simple, green, and natural ways of synthesizing silver nanoparticles out of $\mathrm{AgNO}_{3}$ have been discovered from plant parts. Silver nanoparticles are produced in an ecofriendly and straightforward way, so they can be used effectively as nanomedicines in a wide variety of human ailments [19]. In addition to having some environment-damaging effects, human health, and more importantly, normal cells, using chemical methods for synthesizing AgNPs presents some inherent disadvantages. Electrostatic attraction and affinity with sulfur proteins cause silver ions to adhere to cellular walls and cytoplasmic membranes, resulting in the disruption of bacterial envelopes by increasing cytoplasmic membrane permeability. For the synthesis of silver NPs, researchers investigated the potential of walnut green husk. Walnut green husk extracted into aqueous solution and used as both a reducing and stabilizing agent and synthesized NPs have been further investigated for their anticancer, antioxidant, and antimicrobial properties [20]. The silver nanoparticles can affect bacterial signal transduction by affecting the phosphorylation of protein substrates and can interfere with tyrosine phosphorylation. Signal transduction disruption leads to apoptosis and multiplication termination of the cell [21]. In a study by Radzig and colleagues, GNPs stabilized with hydrolyzed case in peptides significantly reduced the growth and the biomass of Gram-negative bacteria [22]. There are several mechanisms associated with reactive oxygen species (ROS) production in nanoparticles, and it has been suggested that nano-silver toxicity may be a result of ROS, such as with free radicals, produced by oxidative damage $[23,24]$. Implementing a structured delivery system can enhance the effects of AgNPs, which have antimicrobial activity as well in 
addition to have antimicrobial and antiretroviral effects [25-29]. Doxorubicin (DOX), a hydrophilic antitumor drug, combined with AgNPs can significantly decrease the growth and proliferation of tumor cell and is thus much more effective than either DOX or AgNPs on their own [30]. Moreover, an adherent silver nanoparticle releases high concentrations of silver ions on a bacteria's surface and chemically binds with the bacterial surface, leading to its membrane destruction [31].

\subsubsection{Gold Nanoparticles}

The application of colloidal gold solutions to cure specific infections also dates back thousands of years. Since gold is among the most biocompatible metals, as well as possesses a variety of beneficial properties, the need for well-engineered AuNPs is growing on account of their unique properties such as being life-compatible, providing a higher surface reactivity, being quite small, having varied shapes, and resisting oxidation readily. For the synthesis of gold NPs, methods used by researchers include the reduction of gold (III) derivatives. One of the gold reduction methods used by the Daniel and Astruc., 2004, for the synthesis of gold NPs was reducing HAuCl4 with citrate in water leading to synthesis of gold NPs [32]. It has been found that the fruit extracts containing organic constituents that are water soluble act as the principal reducing and capping agents, thereby reducing gold ions and forming AuNPs [33,34]. Antimicrobial properties are contributed by these NPs [35]. There are numerous biomedical applications for AuNPs due to their ability to be biosynthesized from plant extracts by reducing gold salt and thus having broad-range applications in biomedicines [36].

\subsubsection{Copper Nanoparticles}

Copper compounds are used to protect crops from a wide range of microbes and fungi, such as pathogens that cause infections by bacteria and fungi, due to their low cost, high protective activity, and low risk of resistance development [37]. Copper nanoparticles have excellent mending properties and are therefore added to lubricating oils as additives to reduce friction or restore worn surfaces [38]. In addition to substituting essential ions and blocking functional groups of proteins, copper is reported to be able to produce hydroperoxide free radicals, inactivate vital enzymes, and modify membrane integrity, thereby working as an antimicrobial agent $[39,40]$. Some fundamental properties of the antioxidant molecules, such as absorbing, neutralizing, or quenching the singlet and triplet oxygen activate the antioxidant system of CuNPs. For the synthesis of copper NPs, copper acetate tetrahydrate was used. A magnetic stirrer was used to dissolve copper acetate tetrahydrate and aqueous plant extract. The solution was boiled resulting in a green-colored paste, which was further heated in a furnace. After heating, black-colored powdered copper NPs were collected. Phytochemicals present on the surface of CuNPs result in the highest antioxidant activity because they contain a variety of bioreductive groups [41,42].

\subsubsection{Platinum Nanoparticles (PtNPs)}

The large surface areas of platinum nanoparticles (PtNPs) and their alloys make them excellent catalytic materials, and they can be used to reduce pollutants and trigger chemical reactions that facilitate the manufacture of chemicals of various kinds [43]. In addition to confirming the efficacy of nanomaterials, PtNPs have significant potential in the medical field. PtNPs in conjunction with low concentrations of anticancer drugs may provide synergistic anticancer activity/tumor reduction, but new strategies are still needed to investigate the subject. Platinum NPs were synthesized by grinding plant parts to make plant extract. This plant extract then added in $\mathrm{H}_{2} \mathrm{PtCL}_{5}$ and put on constant magnetic stirring. Further, this solution was centrifuged, and platinum NPs were collected [44]. Research conducted by Kim et al., 2008 [45] demonstrates the antioxidant properties of platinum nanoparticles as well as their ability to mimic superoxide dismutase (SOD) and their anti-aging properties in Caenorhabditis elegans. Furthermore, the use of PtNPs as oxidative stress-related 
diseases therapies has been demonstrated in a study in which the addition of PtNPs to hydrogen-dissolved water inhibited tongue carcinoma cells HSC-4 [46].

\subsubsection{Metal Oxide Nanoparticles (MO-NPs)}

The metallic oxides have physiochemical qualities with a broad range of applications in research work and electronics $[47,48]$. In the past decades, metal oxide nanoparticles (MO-NPs) have been broadly used in biomedical applications. MO-NPs are of various types, viz., iron oxide $\left(\mathrm{Fe}_{3} \mathrm{O}_{4}\right)$, copper oxide $(\mathrm{CuO})$, titanium oxide $\left(\mathrm{TiO}_{2}\right)$, and zinc oxide $(\mathrm{ZnO})$. These MO-NPs have a wide range of applications from medical to environmental aspects; they act as a catalyst that can reduce or eliminate toxic impurities or hazardous material present in the environment $[49,50]$. These MO-NPs are usually seen as impervious to the environment and humans [51-53]. MO-NPs damage the bacteria's protein and DNA by inducing oxidative stress for the generation of ROS [54].

\subsubsection{Zinc Oxide Nanoparticles (ZnO-NPs)}

The characteristics of ZnO-NPs lie in their nano size and surface area-to-volume ratio, and personal peculiar biological properties, and they have been recognized and registered as a 'Safe material' [55,56]. A study conducted by [57] shows ZnO-NPs anticancer activity against liver cancer cell line HepG2 with an elevated level of genotoxicity and cytotoxicity associated with cell death/apoptosis. ZnO-NPs (spherical, 96-115 nm size) also exhibit remarkable anti-inflammatory properties with a $66.78 \mu \mathrm{g} / \mathrm{mL}$ IC50 value [58]. The ZnO-NPs have their application in the bioimaging field; they have blue emission and near UV emission spectra with yellow or green luminescence towards oxygen vacancy presence [59-61]. Research reported by Hameed et al., 2019 showed that a combination of NPs (Ag-ZnO-NPs) probably gradually shows greater antimicrobial activity than ZnO-NPs [62]. Probably the most prevalent form of zinc nanoparticles is zinc oxide; this NPs is commonly used in plastic, glass, ceramics, cement, rubber, food supplements, and non-flammable materials due to its wide range of compatible properties, which is also related to its convenient availability and low price [63]. In addition, zinc nanoparticles are both antimicrobial and antibacterial, and are UV-blocking as well, which allows them to be added into fabrics to provide a variety of functions, such as ultraviolet and visible light protection, antibacterial properties, and deodorant functions [64]. In many zinc nanoparticles, fluorescent properties are possible if these NPs can be modified, including ZinS and ZinSe, or quantum dots $\mathrm{CdSe} / \mathrm{ZnS}$, and that is why they are being considered for use in the future. According to a research study, zinc oxide nanoparticles are the most toxic to microbes of all metal oxide nanoparticles that have been examined in vitro for potential toxicity against salmonella typhi and staphylococcus aureus [65]. Moreover, $\mathrm{ZnO}$ nanoparticles have drawn tremendous attention as a low-cost and low-toxicity nanomaterial, with applications in cancer therapy, anti-bacterial properties, anti-inflammatory activities, anti-diabetic properties, and drug delivery $[59,66]$. Zinc oxide NPs were synthesized with plant leaf extract by zinc acetate dihydrate. Firstly, zinc acetate dihydrate was added in distilled water and put into constant stirring, and then aqueous leaf extract was introduced in its solution. After stirring, light yellow-white precipitate was collected and washed with distilled water many times. After distilled water washing, an ethanol wash was performed to remove impurities and the result was precipitate dried in a vacuum to get zinc oxide NPs [67].

\subsubsection{Copper Oxide Nanoparticles (CuO-NPs)}

The unique chemical, physical, electrical, thermal, and biological properties of $\mathrm{CuO}$ $\mathrm{NPs}$ have given them an increased interest as nanomaterials. Copper NPs were synthesized with the help of $\mathrm{Cu}\left(\mathrm{CH}_{2} \mathrm{COO}\right)_{2} \cdot \mathrm{H}_{2} \mathrm{O} \cdot \mathrm{Cu}\left(\mathrm{CH}_{2} \mathrm{COO}\right)_{2} \cdot \mathrm{H}_{2} \mathrm{O}$ was dissolved in distilled water under constant stirring. Plant leaf extract then added in this solution and mixture gains stirred for a few hours. The prepared NPs were centrifuged, collected, and dried in an oven $[68,69]$. Playing a chief role in solar cells, catalysis, lithium-ion battery, and antimicrobial drugs, CuO-NPs are substantial, durable, and, compared to other organic 
and inorganic materials because they ensure a long shelf life $[59,60,70,71]$. Compared with plant extracts, the CuO-NPs are more sensitive to bacterial strains and are lethal due to subsequent interactions with DNA molecules, which cause disorder in structures by cross linking between nucleic acids. $\mathrm{CuO}-\mathrm{NPs}$ are even good antioxidant agents compared to plant extracts [72].

\subsubsection{Iron Oxide Nanoparticles $\left(\mathrm{Fe}_{3} \mathrm{O}_{4}\right.$-NPs)}

Iron Oxide NPs are considered incredibly stellar, biocompatible, and propitious and possess magnetic attributes that make them suitable for treating various diseases and infections [73,74]. A study reported by Chauhan and Upadhyay, 2019 [75] shows that iron oxide NPs were functionalized by coated with L-tyrosine, which gives a significant result against both the pathogenic bacterial strains Staphylococcus typhimurium and Staphylococcus aureus. Iron oxide NPs were synthesized by dissolving henna extract into ferrous sulfate salt solution. Ferrous sulphate was reduced by henna extract and washed with salt solution at constant stirring. The solution turned black, indicating the formation of iron oxide NPs. The solution was cooled and centrifuged to collect pellets. Obtained pellets were washed with distilled water and methanol to remove henna extract impurities and dried overnight to obtain iron oxide NPs. The iron oxide NPs are clinically approved and used as a delegate for magnetic NPs hyperthermia (cancer therapy, to raise the local temperature around the tumor to animate or kill cancer cells) [76]. $\mathrm{Fe}_{3} \mathrm{O}_{4}-\mathrm{NPs}$ as nanomedicine from plant extract (Euphorbia) show prominent antimicrobial activity against Aspergillus niger, Aspergillus fumigatus, and Arthogrophis cuboidal [77]

\subsubsection{Titanium Oxide Nanoparticles $\left(\mathrm{TiO}_{2}-\mathrm{NPs}\right)$}

$\mathrm{TiO}_{2}$-NPs have marvelous morphology, surface chemistry, optical properties, and biocompatibility [78]. They have a wide range of biomedical applications such as in disease diagnosis, surgical tools, bioimaging [79], tissue engineering, drug delivery, and also the synthesis of textiles, cosmetics, plastics, and foodstuffs $[80,81]$. $\mathrm{TiO}_{2}-\mathrm{NPs}_{\text {are }}$ effective against various infectious microorganisms such as bacterial strains, protozoans, algae, viruses, prions, and microbial toxins [82]. $\mathrm{TiO}_{2}-\mathrm{NPs}_{\text {are }}$ used as a nanocarrier (drug delivery) against the MDR breast cancer cells [83]. The green synthesized $\mathrm{TiO}_{2}-\mathrm{NPs}_{\text {are }}$ also found effective against many larvicidal species of parasites such as Catharanthus [84], Calotropis gigantic (L.) [85], Solanum trilobatum L. [86], and Aspergillus niger [87]. The plantderived synthesis of titanium oxide NPs was achieved by mixing $\mathrm{TiO} 2$ with plant extract and incubation at room temperature for $24 \mathrm{~h}$. Green-colored titanium oxide NPs were formed and were collected for further use [88].

\section{Biomedical Applications of Various Plants-Derived NPs}

By reformulating drugs with smaller particle sizes, nanotechnology can boost their oral bioavailability to a greater extent; thus, nanotechnology can replace many conventional oral formulations that are ineffective because they have poor bioavailability as a therapeutic option $[89,90]$. NPs are a promising option for synthetic chemical fungicides, since they provide a more eco-friendly alternative that enhances drug delivery, slowly releases active ingredients, and allows for lower doses of the active ingredient. In addition to being safer and more comfortable for patients, novel drug delivery systems would improve pharmacokinetics for easily degraded peptides and proteins that quickly degrade in the body and decrease the toxicological effects of drugs. Recent studies reported that grape exosome-like nanoparticles administered via oral administration to mice led to cells proliferating in the epithelium of the intestine and caused intestinal stem cells to proliferate. The natural and nontoxic delivery system might therefore be plant-derived edibles composed of nanoparticles characterized by its natural targeting of colonic tissues and anti-inflammatory properties. This system can be used for the treating of digestive tract diseases such as intestinal bowel disease (IBD) [91]. Furthermore, the study of NPs in leukocytes, in order to determine its specific role in gut inflammation, has shown that they 
inhibit Cyclin D1, a molecule that regulates cell cycle. In another study, it was found that in low concentrations, metal oxide nanoparticles have significant antimicrobial activity, and in comparison, with their bulk counterparts, they are low or non-toxic to humans [92]. The growing demand for green chemistry and nanotechnology has pushed for the development of green synthetic methods for the production of nanomaterials using plants, microbes, and other natural resources. Catalysis, medicine, cosmetics, agriculture, food packaging, water treatment, dye degradation, textile engineering, bioengineering sciences, sensors, imaging, biotechnology, electronics, optics, and other biological sectors are just some of the potential applications of these green plant-based NPs [93]. The type and biomedical application of various NPs derived from different kinds of plants are shown in Table 1.

Table 1. Various biomedical applications of various plants-derived NPs.

\begin{tabular}{|c|c|c|c|c|c|c|}
\hline S. No. & Source Plant & $\begin{array}{l}\text { Type of NPs } \\
\text { Prepared }\end{array}$ & Color of NPs & $\begin{array}{l}\text { Phase of } \\
\text { NPs }\end{array}$ & Biomedical Application & Reference \\
\hline \multirow{3}{*}{1.} & Moringa oleifera & $\begin{array}{l}\text { Poly Vinyl } \\
\text { Alcohol-Silver } \\
\text { Nanoparticles } \\
\text { (PVA-AgNPs) }\end{array}$ & Green black & Liquid & $\begin{array}{l}\text { Cervical cancer cell line (HeLa) } \\
\text { proliferation was halted by newly } \\
\text { formed drug-loaded PVA-AgNp } \\
\text { and developed an antineoplastic } \\
\text { (drug that stopped cellular } \\
\text { proliferation, i.e., by inhibiting DNA } \\
\text { synthesis) compound. }\end{array}$ & {$[94,95]$} \\
\hline & $\begin{array}{l}\text { Moringa oleifera } \\
\text { Lam. }\end{array}$ & $\begin{array}{l}\text { Tungsten } \\
\text { Nanoparticles } \\
\text { (W-NPs) }\end{array}$ & Dark brown & Liquid & $\begin{array}{c}\text { It shows antibacterial, antifungal } \\
\text { activity against Bacillus subtilis and } \\
\text { Fusarium exosporium, respectively. } \\
\text { It shows remarkable cytotoxicity } \\
\text { against MCF-7 (breast cancer cell } \\
\text { line) and } 3 \mathrm{~T} 3 \text { (fibroblast cell line). } \\
\text { NPs were potent at } 200 \mu \mathrm{g} / \mathrm{mL} \text { and } \\
0 \mu \mathrm{g} / \mathrm{mL} \text { concentrations, } \\
\text { respectively. }\end{array}$ & [96] \\
\hline & Moringa oleifera & $\begin{array}{l}\text { Titanium } \\
\text { dioxide } \\
\text { nanoparticles } \\
\text { (TiNPs) }\end{array}$ & Brown color & Liquid & $\begin{array}{c}\text { In an animal model (excision } \\
\text { wound model), TiNPs of Moringa } \\
\text { oleifera showedenhancedcontactions } \\
\text { in wound compared to any standard } \\
\text { animal excision model in } \\
\text { the experiment. }\end{array}$ & [97] \\
\hline 2. & $\begin{array}{l}\text { Cyperus } \\
\text { rotundus }(\mathrm{CR})\end{array}$ & $\begin{array}{c}\text { Gold } \\
\text { Nanoparticles } \\
\text { (Au-NPs) }\end{array}$ & Grayish pink & Liquid & $\begin{array}{c}\text { CRnp (Cyperus rotundus gold } \\
\text { nanoparticles) shows antibacterial } \\
\text { activity against various } \\
\text { Gram-positive }\left(\mathrm{G}^{+}\right) \text {(Staphylococcus } \\
\text { aureus, Bacillus subtilis) } \\
\text { and Gram-negative }\left(\mathrm{G}^{-}\right) \\
\text {(Salmonella paratyphi, } \\
\text { Escherichia coli) bacterial species. }\end{array}$ & [98] \\
\hline 3. & $\begin{array}{l}\text { Hibiscus } \\
\text { rosa-Sinensis }\end{array}$ & $\begin{array}{l}\text { Gold } \\
\text { Nanoparticles } \\
\text { (Au-NPs) }\end{array}$ & $\begin{array}{l}\text { Ruby red } \\
\text { color }\end{array}$ & Liquid & $\begin{array}{l}\text { It is nontoxic, highly stable and } \\
\text { therapeutic, and diagnostic } \\
\text { purposes can be used for biomedical } \\
\text { and sensor applications. }\end{array}$ & [99] \\
\hline 4. & $\begin{array}{l}\text { Morus alba } \\
\quad \text { (white } \\
\text { mulberry) }\end{array}$ & $\begin{array}{l}\text { Gold } \\
\text { Nanoparticles } \\
\text { (Au-NPs) }\end{array}$ & $\begin{array}{l}\text { Purple, pink } \\
\text { color }\end{array}$ & Liquid & $\begin{array}{c}\text { It shows inhibition against human } \\
\text { pathogens, i.e., Vibrio cholera }\left(\mathrm{G}^{-}\right) \\
\text {and Staphylococcus aureus }\left(\mathrm{G}^{+}\right) \\
\text {bacteria. }\end{array}$ & [100] \\
\hline 5. & $\begin{array}{l}\text { Catharanthus } \\
\text { roseus Linn. G. }\end{array}$ & $\begin{array}{c}\text { Silver } \\
\text { Nanoparticles } \\
\text { (Ag-NPs) }\end{array}$ & $\begin{array}{l}\text { Dark } \\
\text { yellowish- } \\
\text { brown }\end{array}$ & Liquid & $\begin{array}{l}\text { It shows anti-plasmodial activity } \\
\text { against } P \text {. falciparum. }\end{array}$ & {$[101,102]$} \\
\hline
\end{tabular}


Table 1. Cont.

\begin{tabular}{|c|c|c|c|c|c|c|}
\hline S. No. & Source Plant & $\begin{array}{l}\text { Type of NPs } \\
\text { Prepared }\end{array}$ & Color of NPs & $\begin{array}{l}\text { Phase of } \\
\text { NPs }\end{array}$ & Biomedical Application & Reference \\
\hline 6. & $\begin{array}{l}\text { Phyllanthus } \\
\text { niruri }\end{array}$ & $\begin{array}{c}\text { Silver } \\
\text { Nanoparticles } \\
\text { (Ag-NPs) }\end{array}$ & Brown yellow & Liquid & $\begin{array}{l}\text { These NPs have mosquitocidal } \\
\text { properties against the dengue vector } \\
\text { Aedes aegypti species. }\end{array}$ & [103] \\
\hline 7. & $\begin{array}{l}\text { Abelmoschus } \\
\text { moschatus }\end{array}$ & $\begin{array}{c}\text { Silver } \\
\text { Nanoparticles } \\
\text { (Ag-NPs) }\end{array}$ & $\begin{array}{l}\text { Brownish- } \\
\text { yellow }\end{array}$ & Liquid & $\begin{array}{c}\text { Shows antimicrobial activity against } \\
\text { P. aeruginosa, S. aureus, and } \\
\text { B. subtilis. }\end{array}$ & [104] \\
\hline \multirow{3}{*}{9.} & $\begin{array}{l}\text { Rhizophora } \\
\text { apiculata }\end{array}$ & $\begin{array}{c}\text { Silver } \\
\text { Nanoparticles } \\
\text { (Ag-NPs) }\end{array}$ & $\begin{array}{l}\text { Yellowish- } \\
\text { brown }\end{array}$ & Liquid & $\begin{array}{l}\text { Synthesized NPs were tested } \\
\text { against osteosarcoma cells (MG-63) } \\
\text { in vitro and showed promising } \\
\text { results against bone cancer cell lines. }\end{array}$ & [105] \\
\hline & $\begin{array}{l}\text { Atropa } \\
\text { acuminata } \\
\text { (Indian } \\
\text { Belladonna) }\end{array}$ & $\begin{array}{c}\text { Silver } \\
\text { Nanoparticles } \\
\text { (Ag-NPs) }\end{array}$ & $\begin{array}{l}\text { Yellowish- } \\
\text { brown }\end{array}$ & Liquid & $\begin{array}{l}\text { (i) Synthesized Ag-NPs effectively } \\
\text { controlled autoantigen production } \\
\text { that occurs during the } \\
\text { inflammation process. } \\
\text { (ii) Biosynthesized AgNPs impart } \\
\text { potent cytotoxicity against the HeLa } \\
\text { (cervical cancer) cell line. }\end{array}$ & [106] \\
\hline & $\begin{array}{c}\text { Atropa } \\
\text { acuminata }\end{array}$ & $\begin{array}{c}\text { Silver } \\
\text { Nanoparticles } \\
\text { (Ag-NPs) }\end{array}$ & $\begin{array}{l}\text { Red wine } \\
\text { colour }\end{array}$ & Liquid & $\begin{array}{l}\text { Ag-NPs of belladone shows the } \\
\text { potential halting of BSA protein } \\
\text { denauturation. Mainly atropine and } \\
\text { apoatropine were major } \\
\text { antiinflammatory } \\
\text { agents / compounds present in it. }\end{array}$ & [2] \\
\hline 10. & $\begin{array}{c}\text { Passiflora } \\
\text { caerulea L. } \\
\text { (Passifloraceae) }\end{array}$ & $\begin{array}{l}\text { Zinc oxide } \\
\text { Nanoparticles } \\
\text { (ZnONPs) }\end{array}$ & Yellow color & Liquid & $\begin{array}{l}\text { It shows antibacterial activity } \\
\text { against infectious disease urinary } \\
\text { tract infection (UTI) pathogens such } \\
\text { asthe B. subtilis, Klebsiella pneumonia, } \\
\text { E. coli, Serratia, and } \\
\text { Streptococcus species. }\end{array}$ & [67] \\
\hline \multirow[t]{2}{*}{11.} & \multirow[t]{2}{*}{ Aloe vera } & \multirow{2}{*}{$\begin{array}{l}\text { Silver } \\
\text { Nanoparticles } \\
\text { (Ag-NPs) }\end{array}$} & \multirow[t]{2}{*}{ Gray color } & \multirow[t]{2}{*}{ Solid ppt. } & $\begin{array}{l}\text { The bactericidal effect was observed } \\
\text { against pathogenic } S \text {. epidermidis } \\
\left(\mathrm{G}^{+}\right) \text {and P. aeruginosa }\left(\mathrm{G}^{-}\right) .\end{array}$ & [107] \\
\hline & & & & & $\begin{array}{l}\text { It also shows antibacterial activity } \\
\text { against Kocuriavarians }\left(\mathrm{G}^{+}\right) .\end{array}$ & {$[108]$} \\
\hline 12. & $\begin{array}{l}\text { Ocimum } \\
\text { sanctum (holy } \\
\text { basil or tulsi) }\end{array}$ & $\begin{array}{l}\text { Silver } \\
\text { Nanoparticles } \\
\text { (Ag-NPs) }\end{array}$ & $\begin{array}{l}\text { Yellow- } \\
\text { brown } \\
\text { color }\end{array}$ & Liquid & $\begin{array}{l}\text { The Ag-NPs show the zone of } \\
\text { inhibition against the flourishing of } \\
\text { E. coli and Staphylococcusaureus } \\
\text { bacterial species. }\end{array}$ & [109] \\
\hline \multirow[b]{2}{*}{13.} & $\begin{array}{l}\text { Pelargonium } \\
\text { graveolens }\end{array}$ & $\begin{array}{c}\text { Silver } \\
\text { Nanoparticles } \\
\text { (Ag-NPs) }\end{array}$ & $\begin{array}{l}\text { Dark } \\
\text { brownish } \\
\text { color }\end{array}$ & Liquid & $\begin{array}{l}\text { It exhibits antifungal activity in } \\
\text { Candida tropicalis and Candida kefir } \\
\text { fungal species. }\end{array}$ & [110] \\
\hline & $\begin{array}{l}\text { Pelargonium } \\
\text { graveolens }\end{array}$ & $\begin{array}{l}\text { Silver } \\
\text { Nanoparticles } \\
\text { (Ag-NPs) }\end{array}$ & $\begin{array}{l}\text { Dark brown } \\
\text { color }\end{array}$ & Liquid & $\begin{array}{l}\text { These Ag-NPs exhibited } \\
\text { antibacterial against Candida } \\
\text { albicans, Candida kefyr Candida } \\
\text { tropicalis, Aspergillus niger,and } \\
\text { Aspergillus flavus and had } \\
\text { fungicidal activity. Theyalso show } \\
\text { good wound healing activity and } \\
\text { can be used in the field of medicine. }\end{array}$ & {$[111]$} \\
\hline
\end{tabular}


Table 1. Cont.

\begin{tabular}{|c|c|c|c|c|c|c|}
\hline S. No. & Source Plant & $\begin{array}{c}\text { Type of NPs } \\
\text { Prepared }\end{array}$ & Color of NPs & $\begin{array}{l}\text { Phase of } \\
\text { NPs }\end{array}$ & Biomedical Application & Reference \\
\hline 14. & $\begin{array}{l}\text { Emblica } \\
\text { Officinalis } \\
\text { (Amla) }\end{array}$ & $\begin{array}{c}\text { Phyto- } \\
\text { fabricated } \\
\text { Selenium } \\
\text { Nanoparticles } \\
\text { (PF-SeNPs) }\end{array}$ & $\begin{array}{l}\text { Brick-red } \\
\text { color }\end{array}$ & Liquid & $\begin{array}{l}\text { It exhibits potent antibacterial } \\
\text { activity towards } \mathrm{G}^{+} \text {bacteria ( } S \text {. } \\
\text { aureus MTCC } 96, \text { E. faecalis MTCC } \\
\text { 439, and L. monocytogenes MTCC } \\
\text { 657) compared to } \mathrm{G}^{-} \text {bacteria (E. coli } \\
\text { MTCC 4). }\end{array}$ & [112] \\
\hline 15. & $\begin{array}{l}\text { Helianthus } \\
\text { annuus } \\
\text { (Sunflower) } \\
\text { seed }\end{array}$ & $\begin{array}{l}\text { Zirconium } \\
\text { oxide } \\
\text { nanoparticles } \\
\left(\mathrm{ZrO}_{2} \mathrm{NPs}\right)\end{array}$ & Black powder & Liquid & $\begin{array}{l}\text { It shows the inhibition of } \mathrm{G} \text { bacteria } \\
\text { (E. coli, P. aeruginosa, and } K \text {. } \\
\text { pneumonia) in comparison to } \mathrm{G}^{+} \\
\text {S. aureus bacteria. }\end{array}$ & [113] \\
\hline 16. & $\begin{array}{c}\text { Papaver } \\
\text { somniferum L. }\end{array}$ & $\begin{array}{l}\text { Lead oxide } \\
(\mathrm{PbO}) \text { and iron } \\
\text { oxide }\left(\mathrm{Fe}_{2} \mathrm{O}_{3}\right) \\
\text { nanoparticles }\end{array}$ & Violet color & Solid pellets & $\begin{array}{l}\text { (i) These Biogenic NPs showed } \\
\text { potency against most of the } \\
\text { pathogenic strains such as } \mathrm{G}^{+} \\
\text {(Bacillus subtilis and Staphylococcus } \\
\text { epidermidis) and } \mathrm{G}^{-} \text {(Klebsiella } \\
\text { pneumonia and Pseudomonas } \\
\text { aeruginosa) bacterial species, while } S \text {. } \\
\text { epidermidis was found prone to } \\
\mathrm{Fe}_{2} \mathrm{O}_{3} \text { NPs. } \\
\text { (ii) It also exhibits an antifungal } \\
\text { tendency against fungal pathogens } \\
\text { such as Aspergillus flavus, Fusarium } \\
\text { solani, Mucormycosis, Aspergillus } \\
\text { niger, and Aspergillus fumigates. } \\
\text { The PbO NPs show that the highest } \\
\text { inhibition was found against } \\
\text { F. solani. } \\
\text { While Fe } \mathrm{O}_{3} \text { NPs also show it, the } \\
\text { highest antifungal characteristics } \\
\text { werefound towards F. solani. } \\
\text { (iii) Cell culture study: Cytotoxicity } \\
\text { screening performedagainst HepG2 } \\
\text { (Human liver cancer) tumorigenic } \\
\text { cell line. PbO NPs showed elevated } \\
\text { cytotoxicity in contrast to } \\
\text { Fe } \mathrm{O}_{3} \text { NPs. }\end{array}$ & [114] \\
\hline 17. & Basella alba $\mathrm{L}$. & $\begin{array}{l}\text { Metallic Silver } \\
\text { Nanoparticles } \\
\text { (Ag-NPs) }\end{array}$ & $\begin{array}{l}\text { Yellowish- } \\
\text { brown } \\
\text { color }\end{array}$ & Liquid & $\begin{array}{c}\text { It shows antibacterial properties } \\
\text { towards pathogens } \mathrm{G}^{+}(\text {S. aureus, } \\
\text { P. aeruginosa) and } \mathrm{G}^{-} \\
\text {(Enterococcus feces, E. coli). } \\
\text { S. aureus and E. coli bacterial species } \\
\text { show a broad region of inhibition. }\end{array}$ & [115] \\
\hline 18. & $\begin{array}{l}\text { Oryza sativa } \\
\text { (Black rice) }\end{array}$ & $\begin{array}{c}\text { Silver } \\
\text { Nanoparticles } \\
\text { (Ag-NPs) }\end{array}$ & $\begin{array}{l}\text { Brownish } \\
\text { color }\end{array}$ & Liquid & $\begin{array}{l}\text { Ag-NPs-loaded samples against } \\
\text { E. coli and S. aureus clearly show } \\
\text { inhibition zones in a bacterial } \\
\text { culture plate. }\end{array}$ & [116] \\
\hline 19. & $\begin{array}{c}\text { Jatropha } \\
\text { curcas(JC) }\end{array}$ & $\begin{array}{c}\text { Silver } \\
\text { Nanoparticles } \\
\text { (Ag-NPs) }\end{array}$ & $\begin{array}{l}\text { Brownish- } \\
\text { gray } \\
\text { color }\end{array}$ & Liquid & $\begin{array}{l}\text { JC-AgNps show action against } \\
\text { Listeria monocytogenes (Food born } \\
\text { pathogen), a bacterial pathogen. }\end{array}$ & [117] \\
\hline 20. & Carica papaya & $\begin{array}{c}\text { Silver } \\
\text { Nanoparticles } \\
\text { (Ag-NPs) }\end{array}$ & Dark brown & Liquid & $\begin{array}{l}\text { It highly inhibited DENV-2 } \\
\text { replication in vitro conditions with } \\
\text { a viral inhibition percentage }>90 \text {. }\end{array}$ & [112] \\
\hline
\end{tabular}


Table 1. Cont.

\begin{tabular}{|c|c|c|c|c|c|c|}
\hline S. No. & Source Plant & $\begin{array}{c}\text { Type of NPs } \\
\text { Prepared }\end{array}$ & Color of NPs & $\begin{array}{c}\text { Phase of } \\
\text { NPs }\end{array}$ & Biomedical Application & Reference \\
\hline 21. & Mangifera indica & $\begin{array}{c}\text { Gold } \\
\text { Nanoparticles } \\
\text { (Au-NPs) }\end{array}$ & $\begin{array}{l}\text { Deep purple } \\
\text { red/ruby red } \\
\text { color. }\end{array}$ & Liquid & $\begin{array}{l}\text { It was found that Au-NPs were } \\
\text { effective at low concentrations when } \\
\text { used against } G^{-}(E . \text { coli) and } G+ \\
\text { (S. aureus) bacterial species. }\end{array}$ & [118] \\
\hline 22. & $\begin{array}{c}\text { P. caerulea L. } \\
\text { (Passifloraceae) }\end{array}$ & $\begin{array}{l}\text { Zinc oxide } \\
\text { Nanoparticle } \\
\text { (ZnO-NPs) }\end{array}$ & Yellow color & Liquid & $\begin{array}{l}\text { It shows antibacterial activity } \\
\text { against urinary tract infection (UTI) } \\
\text { pathogens such as Streptococcus sp., } \\
\text { Serratia sp., E. coli, Klebsiella } \\
\text { pneumonia, and B. subtilis. }\end{array}$ & [67] \\
\hline 23. & $\begin{array}{c}\text { Momordica } \\
\text { charantia }\end{array}$ & $\begin{array}{l}\text { Copper Oxide } \\
\text { Nanoparticles } \\
\text { (CuO-NPs) }\end{array}$ & Brown color & Liquid & $\begin{array}{l}\text { CuO-NPs were effective against } \\
\text { Multi-Drug Resistance (MDR) } \\
\text { bacterial strains such as } \mathrm{G}^{+} \text {bacteria } \\
\text { Bacillus cereus, Streptococcus mutans, } \\
\text { Streptococcus viridans, etc., and } \mathrm{G}^{-} \\
\text {bacteria such as Proteus Vulgaris, } \\
\text { Escherichia coli, and } \\
\text { Pseudomonas aeruginosa. }\end{array}$ & [119] \\
\hline 24. & $\begin{array}{l}\text { Berberis lycium } \\
\text { ROYLE(SU) }\end{array}$ & $\begin{array}{l}\text { Copper Oxide } \\
\text { Nanoparticle } \\
\text { (CuO-NPs) }\end{array}$ & Brown color & Liquid & $\begin{array}{l}\text { Its NPs are sensitive against the } \mathrm{G}^{+} \\
\text {and } \mathrm{G}^{-} \text {bacterial sp. The highest } \\
\text { zone of inhibition in the case of } \mathrm{G}^{+} \\
\text {bacteria is towards Streptococcus } \\
\text { Mutants, while in the case of } \mathrm{G}^{-} \\
\text {bacteria, it is towards Escherichia coli. }\end{array}$ & [72] \\
\hline 25. & $\begin{array}{l}\text { Zanthoxylum } \\
\text { armatum DC. }\end{array}$ & $\begin{array}{l}\text { Copper Oxide } \\
\text { Nanoparticle } \\
\text { (CuO-NPs) }\end{array}$ & Black color & Liquid & $\begin{array}{l}\text { It shows antibacterial activity and } \\
\text { maximum inhibition against } \\
\text { Streptococcus mutans }\left(\mathrm{G}^{+}\right) \text {bacterium, } \\
\text { while in case of } \mathrm{G}^{-} \text {bacterium it } \\
\text { shows maximum inhibition against } \\
\text { Pseudomonas aeruginosa. }\end{array}$ & [72] \\
\hline
\end{tabular}

\section{Nanomedicine as Promising Anticancer Agent}

In carrying anticancer agents, nanoparticles are primarily advantageous because of their ability to deliver anticancer agents to the tumor, tumor imaging, the storage of thousands of molecules of drugs, and resistance to solubility, stability, and stability issues [120]. In a study, AuNPs are found to have anticancerous activity against many cell lines such as the K-562 cell line, Hela cell line, MDA-MB cell line, and A-549 cell line, but surprisingly there was no effect of these AuNPs seen against the Vero cell line [121]. The $\mathrm{ZnO}$ nanoparticles present some cytotoxicity in cancer cells primarily through the increased release of dissolved zinc ions inside the cell, with enhanced ROS production leading to apoptotic cell death [122]. In a study, [123] fabricated $\mathrm{ZnO}$ nanoparticles were used for an anticancer evaluation using a chemical precipitation method. They demonstrated that differently sized ZnO nanoparticles inhibited fibrosarcoma HT1080 cell proliferation. In a study, the potential mechanism of $\mathrm{TiO}_{2}$ (titanium dioxide) nanoformulations inhibiting cell proliferation was demonstrated. Studies have shown that $\mathrm{TiO}_{2}$ can disrupt the cell cycle by blocking a number of DNA checkpoints during cell division [124]. Other than their studies in tumor microenvironments, TiO2-NPs have been involved in the redox homeostasis, neurodegeneration pathways, and NAD salvage mechanisms [125]. According to many recent studies, it was found that pure $\mathrm{Au}-\mathrm{TiO}_{2}-\mathrm{NPs}, \mathrm{Zn}-\mathrm{TiO}_{2}-\mathrm{NPs}$, and $\mathrm{Ag}-\mathrm{TiO}_{2}-\mathrm{NPs}_{\text {are }}$ capable of becoming genotoxic drugs when used as nanomedicines or chemotherapeutic drugs to treat cancer. Additionally, the radioactive probes can be used to penetrate deep into cancer tissues to treat them more effectively [126,127]. According to a study, nanomedicine 
must be goes through five steps to reach tumor cells including circulation, accumulation, penetration, and internalization followed by release [128].

\section{Plant-Derived Nanostructures and Their Medicinal Applications}

The nanoparticles and nanostructures have been widely used in the field of biomedical sciences, drug-gene delivery systems, electronics, mechanics, catalysis, energy science, etc. $[129,130]$. The plant's secondary metabolite, like polyphenols, has shown excellent effects, acting as an antimicrobials, antioxidants, and modern biomedicine [131,132]. Plants can produce a large type of typical nanostructures as matching with the current material (nanostructure) produced by the synthetic, engineered process [133]. Various types of plant-derived nanostructures serve as natural nano-factories of medicinal NPs, such as polysaccharide-based NPs [134-136], protein-based NPs [137-139], carbon-based nanostructures [140-142], adhesive NPs [143], exosome-like NPs [91,144], lipid-based NPs [145,146], silica NPs [147], etc. Various biomedical applications of plant-derived nanostructures are mentioned in Figure 3.

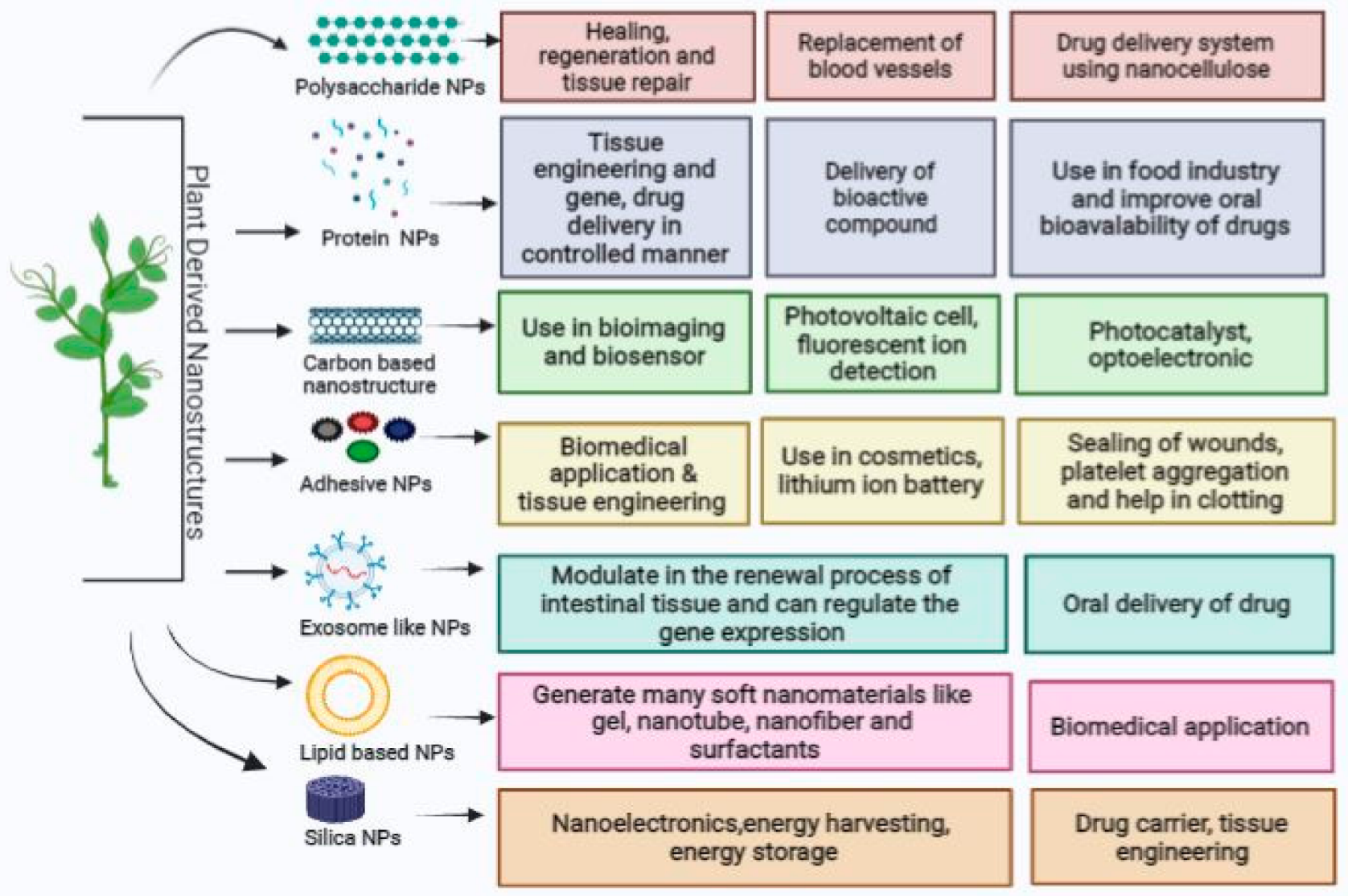

Plant derived nanostructure and their application in biomedical field

Figure 3. Plant-derived nanostructure and their application in biomedical field.

\section{Plant-Derived Nanofibers and Their Role in Wound Healing}

Nanofibers have characteristic features such as a porous structure, exquisite interconnectivity between pores (desirable for wound healing), moisture availability, oxygen permeability, and the inhibitory effect against the microorganisms from outside; they have compliance to the skin at the wound site and have an awful property to slacken skin scars [148-150]. Many natural biopolymers such as polysaccharides (cellulose, starch, chitin, and chitosan), proteins (gelatin, keratin, fibrin, silk fibroin, and zein), proteoglycans, bacterial and algal polysaccharides, plant gums, and bioactive compounds (phytochemical constituents) have been at the center of attraction in the biomedical field. These natural 
plant-derived biopolymers have played a significant role in wound dressing and been successfully exploited for the formation of nanofibers through electrospinning. They show biocompatibility with materials required for wound healing due to similarity with human macromolecules, having properties such as biodegradability, a hemostatic nature, and non-toxicity [151-155]. Electrospun nanofibrous wound dressings materials loaded with synthetic antibiotics and nanoparticles have a detrimental effect on the environment and human health. Nanoparticles-loaded with phytochemicals have fewer side effects, are readily available, and are less costly [156]. A variety of natural biomolecules or compounds are selected for the nanoparticles. These nanoparticles are then converted into a nanofiber form that efficiently helps in wound dressing. The process of nanofiber formation and wound healing is illustrated in Figure 4. Curcuma longa $\mathrm{L}$ is the first plant extract loaded with ultra-fine cellulose acetate fiber mats and was manufactured by electrospinning [157]. The active component of Curcuma longa $\mathrm{L}$ is curcumin, which possesses a significant range of biological activities such as antibacterial, anti-inflammatory, antimicrobial, antioxidant, angiogenic effect, and anticancerous properties [158].

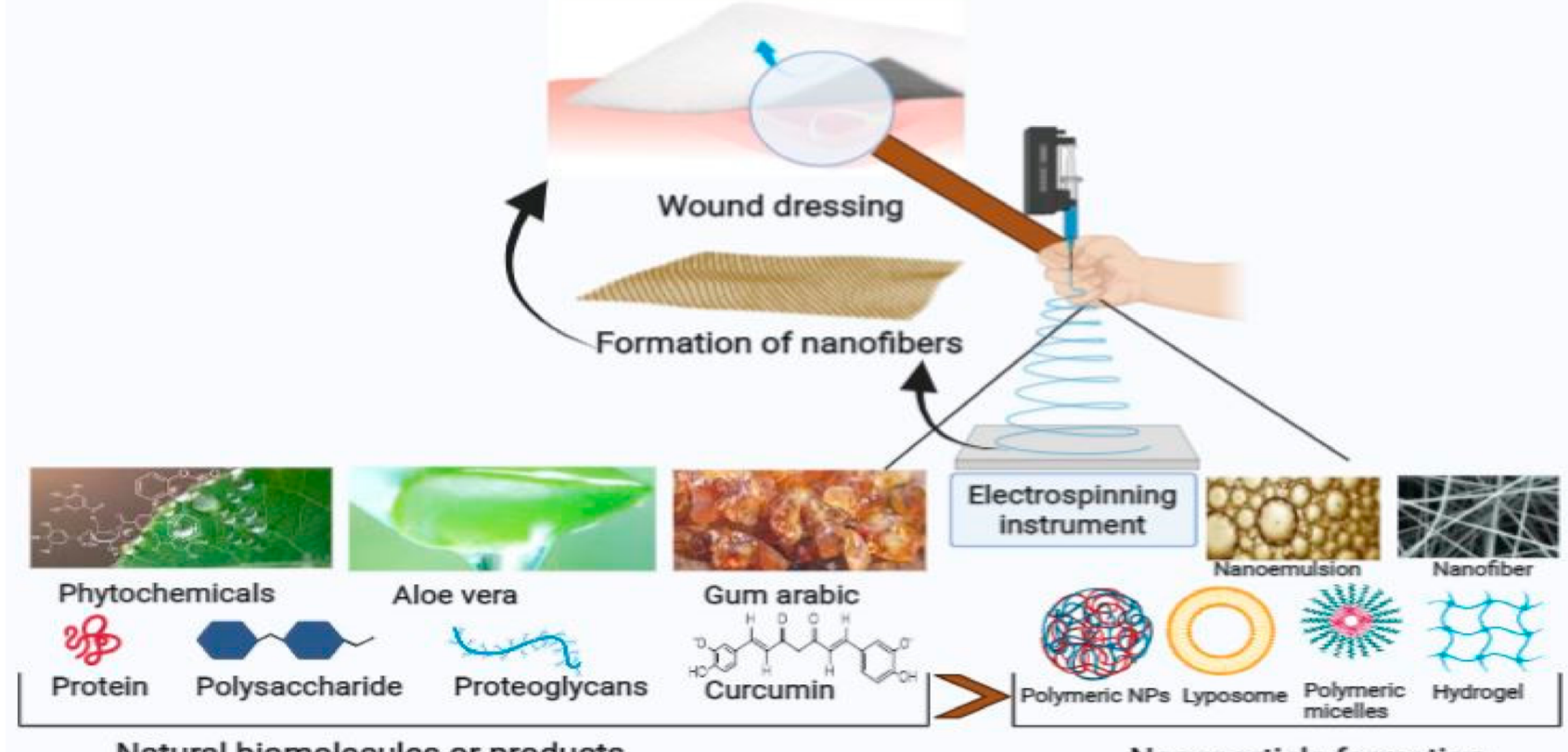

Natural biomolecules or products

Nanoparticle formation

\section{Formation of nanofibers and wound healing (dressing) mechanism}

Figure 4. Schematic illustration of nanofiber formation and their role in wound healing.

\section{Plant-Derived Nanostructure Biomaterial and Their Role in Bone Regeneration}

The process of bone regeneration occurs synergistically; a small defect in bone leads to the action of different cells, i.e., mesenchymal cells, osteogenic cells, and the cells of the immune system. Under the process of self-repaired bone leads to some physio-chemical and mechanical properties to tautologize the replaced bone [159]. Plant polyphenols show a promising role in tissue regeneration, as the process of remodeling is exacting a sophisticated process for the development of bone tissue regeneration. Due to natural antimicrobial and antioxidant activity, polyphenol acts as a potential drug in the modern biomedicine world. Polyphenols also have numerous bioactive compounds, micronutrients that maintain and protect bone health $[160,161]$. Electrospinning is widely used techniques that can produce nano-pore size and nano-diameters of plant-derived polyphenols with the fabrication of nano-fibrous scaffolds, as shown in Figure 5. Nanofibers created with electrospinning mimic the extracellular matrix (ECM) closely and have the same porosity, mechanical property, surface area-to-volume ratio (supports increased cell adhesion), pro- 
liferation, growth, and spreading [147]. The natural composition of bone is approximately 60 percent (dry weight) with the nanostructured composite having minerals such as calcium phosphate $(\mathrm{CaP})$. The bone tissue regeneration process through the electrospinning method containing photo-bioactive (polyphenol) nanofiber formation is shown in Figure 5.

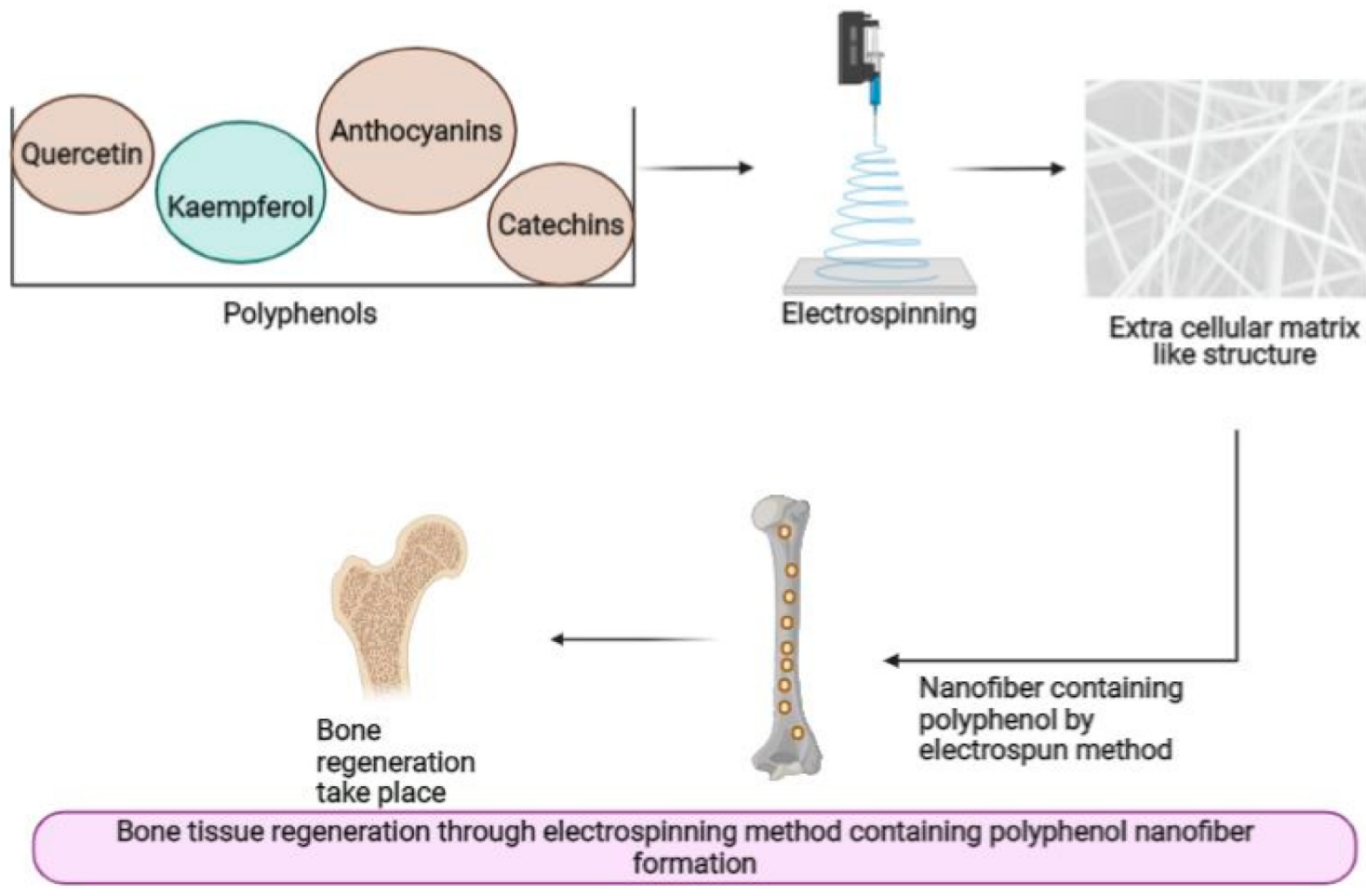

Figure 5. Schematic representation of bone tissue regeneration process through electrospinning method containing photo-bioactive (polyphenol) nanofiber formation.

\section{Plant-Based Nanoparticles (PBNPs) as a Promising Nanomedicine against COVID-19}

Severe acute respiratory syndrome coronavirus 2 (SARS-CoV-2) causes COVID-19 (coronavirus disease 2019). The virus attachment to the host cell occurs with the viral spike (S) protein, and it also serves as the impassable pursuit for drug development against this deadly virus [162]. Plants contain natural products such as flavonoids, alkaloids, steroids, saponins, tannins, coenzymes, etc., that are present in abundance and are found in different parts of a plant such as the leaves, flowers, roots, stem, shoots, bark, and fruits as well in seeds. These phytochemicals are known as secondary metabolites, acting as a stabilizing and reducing agent for bio-reduction reactions to form novel therapeutics such as metallic nanoparticles (MNPs) [163]. PBNPs provide an alternative method for the antipathogenic property towards microorganisms (virus, fungi, bacteria, yeast) through reactive oxygen species (ROS) generation by the process of photocatalysis. The PBNPs can be used as a targeted drug delivery agent for the pulmonary system and can halt the interaction between the receptor (angiotensin-converting enzyme 2) and viral S protein. A recent study reported by Medicago and GSK showed a positive phase 3 trial of the PBNPs-derived vaccine against COVID-19. Medicago's reported plant-based NPs which was developed by using bizarre technology to make virus-like particles (VLPs) for its protein vaccines. These VLPs generally mimic the structure of the lethal, native structure of the virus, hence allowing the immune system to recognize it easily [164]. Additionally, another study showed that plant-derived silica NPs present on a mask minimizes the SARS-Cov-2 infectivity to zero [165]. 


\section{Advantages and Disadvantages of Plant-Derived NPs}

Plant-derived NPs are of significant use in the medicinal field, having biomedical applications. These NPs have a convenient shape and size and can be used for non-toxic and efficient drug delivery, anticancer treatment, wound healing, bone regeneration, and much more. NPs synthesis methods from plants are very novel and cost efficient, having no harm to nature or the environment. Due to the very tiny size, NPs can penetrate small capillaries, and loaded drugs can be efficiently absorbed into cells, allowing them to accumulate at their target sites. Moreover, the drug is retained at the active site for a longer period of time. It is possible to control the size of nanoparticles used in drug delivery and provide good protection for the drug. Apart from various advantages of plant-derived NPs, there are some disadvantages that need to be discussed. By inhaling air with nano particles in it for a few seconds, nanoparticles can cause inhalation problems, and other fatal diseases. As a result of their small sizes, NPs may also damage the lungs if inhaled for a prolonged period of time. Inhibition of autonomic equilibrium by nanoparticles directly impacting the heart and blood vessels [166]. Although plant-derived NPs are of significant interest, still, some methods that require chemicals are expensive and can also become toxic.

\section{Discussion and Conclusions}

Many researchers have a great deal of interest in the use of nanotechnology to create materials that are diverse in composition. Because nanotechnology generates materials with multi-dimensional structures typically controlled on the nanoscale range, applications of nanotechnology in medicine have sparked vast interest in cancer treatment wound healing, bone regeneration, and diagnosis. If more effective tumor-targeting strategies can be developed, nanoparticles will certainly play a more prominent role in cancer chemotherapy. In the biomedical field, nanoparticles with antimicrobial properties are widely used, and using secondary metabolites of plants for their synthesis and stabilization can substantially increase the effectiveness of metal and metal-oxide nanoparticles against microbes, in addition to making them biocompatible. For widespread medical use, the toxicity assessment of metal nanoparticles is imperative. Herbal extracts (due to the presence of abundant phytochemicals such as flavonoids, alkaloids, tannins, saponins, and other metabolites) serve as potential means for the production of nanomaterials via safer pathways. Various secondary metabolites embodying the extracts play the roles of stabilizing and/or reducing agents to restructure the nanoparticles (NPs) to have diverse applications in assorted fields, but predominantly in nanomedicine. Plant-derived NPs are even highly potent against the widely spread deadly disease COVID-19. In this way, the green initiatives of NPs synthesis are becoming more admired, and the topic anticipated to develop exponentially in the days ahead.

Author Contributions: Conceptualization, R.T. and T.K.U.; methodology, R.T., T.K.U. and A.B.S.; software and validation, M.H.M., F.K. and P.P.; investigation and formal analysis; T.K.U., A.B.S., F.K., P.P., K.M., N.N., A.H., N.M.A., S.A., S.S. and M.S.; resources, T.K.U. and A.B.S.; data curation, T.K.U., M.S., A.B.S., writing - original draft preparation, R.T., T.K.U. and A.B.S.; writing review and editing, T.K.U., A.B.S., F.K., P.P., K.M., N.N., A.H., N.M.A., S.A., S.S. and M.S.; visualization, T.K.U. and A.B.S.; supervision, T.K.U., A.B.S. and M.S.; project administration, T.K.U.; funding acquisition, K.M., N.N., A.H., N.M.A., S.A., S.S. and M.S. All authors have read and agreed to the published version of the manuscript.

Funding: The authors extend their appreciation to the Centre of Research for Development, Parul University for providing the Intramural research grant and are also thankful to the Deanship of Scientific Research at King Khalid University for funding this work through a small Research Group Project under grant number RGP.01-345-42.

Institutional Review Board Statement: Peer reviewers on this manuscript have no relevant financial or other relationships to disclose.

Informed Consent Statement: Not applicable. 


\section{Data Availability Statement: Not applicable.}

Acknowledgments: The authors are very grateful to Medical, G.M.P., Centre of Research for Development (CR4D), Parul University, V.G., India for providing the facility during the compilation of ideas in the form of a manuscript. The authors also extend thanks to the Deanship of Scientific Research at King Khalid University for funding this work through a Group Research Project under grant number RGP.01-345-42.

Conflicts of Interest: No affiliations or financial involvements by the authors are related to any organization or entity with a financial interest in or financial conflict with the subject matter or materials in the manuscript, other than those disclosed.

\section{References}

1. Salam, H.A.; Rajiv, P.; Kamaraj, M.; Jagadeeswaran, P.; Gunalan, S.; Sivaraj, R. Plants: Green route for nanoparticle synthesis. Int. J. Biol. Sci. 2012, 1, 85-90.

2. Elangovan, K.; Elumalai, D.; Anupriya, S. Phyto mediated biogenic synthesis of silver nanoparticles using leaf extract of Andrographisechioides and its bio-efficacy on anticancer and antibacterial activities. J. Photochem. Photobiol. B Biol. 2015, 151, 118-124. [CrossRef] [PubMed]

3. Iravani, S. Green synthesis of metal nanoparticles using plants. GreenChem 2011, 13, 2638-2650. [CrossRef]

4. Singh, J.; Dutta, T.; Kim, K.H.; Rawat, M.; Samddar, P.; Kumar, P. 'Green' synthesis of metals and their oxide nanoparticles: Applications for environmental remediation. J. Nanobiotechnol. 2018, 16, 1-24. [CrossRef]

5. Lengke, M.F.; Sanpawanitchakit, C.; Southam, G. Biosynthesis of Gold Nanoparticles: A Review. In Metal Nanoparticles in Microbiology, 1st ed.; Duran, R.N., Ed.; Springer: New York, NY, USA, 2011; pp. 37-74.

6. Dahoumane, S.A.; Jeffryes, C.; Mechouet, M.; Agathos, S.N. Biosynthesis of inorganic nanoparticles: A fresh look at the control of shape, size and composition. Bioengineering 2017, 4, 14. [CrossRef]

7. Vimbela, G.V.; Ngo, S.M.; Fraze, C.; Yang, L.; Stout, D.A. Antibacterial properties and toxicity from metallic nanomaterials. Int. J. Nanomed. 2017, 12, 3941. [CrossRef]

8. Holister, P.; Weener, J.W.; Vas, C.R.; Harper, T. Nanoparticles, Technology White Papers nr. 3. Cientifica $2003,3,1-11$.

9. Wang, W.; Chen, Q.; Jiang, C.; Yang, D.; Liu, X.; Xu, S. One-step synthesis of biocompatible gold nanoparticles using gallic acid in the presence of poly-(N-vinyl- 2-pyrrolidone). Colloids Surf. A Physicochem. Eng. Aspects 2007, 301, 73-79. [CrossRef]

10. Schmid, G.; Simon, U. Gold nanoparticles: Assembly and electrical properties in 1-3 dimensions. Chem. Commun. 2005, 6, 697-710. [CrossRef]

11. Solanki, A.; Kim, J.D.; Lee, K.B. Nanotechnology for regenerative medicine: Nanomaterials for stem cell imaging. Nanomedicine 2008, 3, 567-578. [CrossRef]

12. Jain, S.K.; Sahni, Y.; Neetu, R.; Vidhi, G. Nanotoxicology: An emerging discipline. Vet. World 2011, 4, 35-40. [CrossRef]

13. Venkatesan, J.; Kim, S.K. Nano-hydroxyapatite composite biomaterials for bone tissue engineering-A review. J. Biomed. Nanotechnol. 2014, 10, 3124-3140. [CrossRef]

14. Yi, H.; Rehman, F.U.; Zhao, C.; Liu, B.; He, N. Recent advances in nano scaffolds for bone repair. Bone Res. 2016, 4, 1-11. [CrossRef]

15. Silva, G.A. Introduction to nanotechnology and its applications to medicine. Surg. Neurol. 2004, 61, 216-220. [CrossRef]

16. Caruthers, S.D.; Wickline, S.A.; Lanza, G.M. Nanotechnological applications in medicine. Curr. Opin. Biotechnol. 2007, 18, 26-30. [CrossRef]

17. Kubik, T.; Bogunia-Kubik, K.; Sugisaka, M. Nanotechnology on duty in medical applications. Curr. Pharm. Biotechnol. 2005, 6, 17-33. [CrossRef]

18. Kayser, O.; Lemke, A.; Hernandez-Trejo, N. The impact of nanobiotechnology on the development of new drug delivery systems. Curr. Pharm. Biotechnol. 2005, 6, 3-5. [CrossRef]

19. Paul, S.; Sasikumar, C.S.; Singh, A.R. Preliminary investigation of synthesizing silver nanoparticles from the different biological source: A modern eco-friendly tool. Int. J. Pharm. Res. 2015, 4, 135-148.

20. Khorrami, S.; Zarrabi, A.; Khaleghi, M.; Danaei, M.; Mozafari, M. Selective cytotoxicity of green synthesized silver nanoparticles against the MCF-7 tumor cell line and their enhanced antioxidant and antimicrobial properties. Int. J. Nanomed. 2018, 13, 8013-8024. [CrossRef]

21. Li, L.; Li, L.; Zhou, X.; Yu, Y.; Li, Z.; Zuo, D.; Wu, Y. Silver nanoparticles induce protective autophagy via Ca ${ }^{2+}$ /CaMKK $\beta$ /AMPK/mTOR pathway in SHSY5Y cells and rat brains. Nanotoxicology 2019, 13, 369-391. [CrossRef]

22. Radzig, M.A.; Nadtochenko, V.A.; Koksharova, O.A.; Kiwi, J.; Lipasova, V.A.; Khmel, I.A. Antibacterial effects of silver nanoparticles on gram-negative bacteria: Influence on the growth and biofilms formation, mechanisms of action. Colloids Surf. $B$ Biointerfaces 2013, 102, 300-306. [CrossRef]

23. Kim, J.S.; Kuk, E.; Yu, K.N.; Kim, J.H.; Park, S.J.; Lee, H.J.; Kim, S.H.; Park, J.K.; Park, Y.H.; Hwang, C.Y.; et al. Antimicrobial effects of silver nanoparticles. Nanomed. Nanotechnol. 2007, 3, 95-101. [CrossRef]

24. Hwang, E.T.; Lee, J.H.; Chae, Y.J.; Kim, Y.S.; Kim, B.C.; Sang, B.I.; Gu, M.B. Analysis of the toxic mode of action of silver nanoparticles using stress-specific bioluminescent bacteria. Small 2008, 4, 746-747. [CrossRef] 
25. Sriram, M.I.; Kanth, S.B.; Kalishwaralal, K.; Gurunathan, S. Antitumor activity of silver nanoparticles in Dalton's lymphoma ascites tumor model. Int. J. Nanomed. 2010, 5, 753-762. [CrossRef]

26. Sankar, R.; Karthik, A.; Prabu, A.; Karthik, S.; Shivashangari, K.S.; Ravikumar, V. Origanum vulgare mediated biosynthesis of silver nanoparticles for its antibacterial and anticancer activity. Colloids Surf. B Biointerfaces 2013, 108, 80-84. [CrossRef]

27. Kathiravan, V.; Ravi, S.; Ashokkumar, S. Synthesis of silver nanoparticles from Melia dubia leaf extract and their in vitro anticancer activity. Spectrochim. Acta Part A Mol. Biomol. Spectrosc. 2014, 130, 116. [CrossRef]

28. Lara, H.H.; Ixtepan-Turrent, L.; Treviño, E.N.; Singh, D.K. Use of silver nanoparticles increased inhibition of cell-associated HIV-1 infection by neutralizing antibodies developed against HIV-1 envelope proteins. J. Nanobiotechnol. 2011, 9, 38. [CrossRef]

29. Galdiero, S.; Falanga, A.; Vitiello, M.; Cantisani, M.; Marra, V.; Galdiero, M. Silver nanoparticles as potential antiviral agents. Molecules 2011, 16, 8894-8918. [CrossRef]

30. Prados, J.; Melguizo, C.; Ortiz, R.; Velez, C.; Alvarez, P.J.; Arias, J.L.; Ruiz, M.A.; Gallardo, V.; Aranega, A. Doxorubicin-loaded nanoparticles: New advances in breast cancer therapy. Anticancer Agents Med. Chem. 2012, 12, 1058-1070. [CrossRef]

31. Li, W.; Calle, L.M.; Hanford, A.J.; Stambaugh, I.; Callahan, M.R. Investigation of silver biocide as a disinfection technology for spacecraft-an early literature review. In Proceedings of the 48th International Conference on Environmental Systems, Albuquerque, NM, USA, 8-12 July 2018; p. ICES-2018-82.

32. Daniel, M.C.; Astruc, D. Gold nanoparticles: Assembly, supramolecular chemistry, quantum-size-related properties, and applications toward biology, catalysis, and nanotechnology. Chem. Rev. 2004, 104, 293-346. [CrossRef] [PubMed]

33. Ghosh, S.K.; Pal, T. The effect of the interaction between particles on the surface of the nanoparticles. Chem. Rev. 2007, 107, 4797-4862. [CrossRef] [PubMed]

34. Shah, M.; Fawcett, D.; Sharma, S.; Tripathy, S.K.; Poinern, G.E. Green synthesis of metallic nanoparticles via biological entities. Materials 2015, 29, 7278-7308. [CrossRef] [PubMed]

35. Fayaz, M.A.; Balaji, K.; Girilal, M.; Yadav, R.; Kalaichelvan, P.T.; Venkatesan, R. Biogenic synthesis of silver nanoparticles and their synergistic effect with antibiotics: A study against gram-positive and gram-negative bacteria. Nanomed. Nanotech. Biol. Med. 2010, 6, 103-109. [CrossRef]

36. Huaizhi, Z.; Yuantao, N. China's ancient gold drugs. Gold Bull. 2001, 34, 24-29. [CrossRef]

37. Keller, A.A.; Adeleye, A.S.; Conway, J.R.; Garner, K.L.; Zhao, L.; Cherr, G.N.; Hong, J.; Gardea-Torresdey, J.L.; Godwin, H.A.; Hanna, S.; et al. Comparative environmental fate and toxicity of copper nanomaterials. NanoImpact 2017, 7, 28-40. [CrossRef]

38. Liu, G.; Li, X.; Qin, B.; Xing, D.; Guo, Y.; Fan, R. Investigation of the mending effect and mechanism of copper nanoparticles on a tribologically stressed surface. Tribol. Lett. 2004, 17, 961-966. [CrossRef]

39. Arjunan, N.; Singaravelu, C.M.; Kulanthaivel, J.; Kandasamy, J. A potential photocatalytic, antimicrobial and anticancer activity of chitosan-copper nanocomposite. Int. J. Biol. Macromol. 2017, 104, 1774-1782. [CrossRef]

40. Al-Enizi, A.M.; Ahamad, T.; Al-Hajji, A.B.; Ahmed, J.; Chaudhary, A.A.; Alshehri, S.M. Cellulose gum and copper nanoparticles based hydrogel as antimicrobial agents against urinary tract infection (UTI) pathogens. Int. J. Biol. Macromol. 2018, 109, 803-809. [CrossRef]

41. Rehana, D.; Mahendiran, D.; Kumar, R.S.; Rahiman, A.K. Evaluation of antioxidant and anticancer activity of copper oxide nanoparticles synthesized using medicinally important plant extracts. Biomed. Pharmacother. 2017, 89, 1067-1077. [CrossRef]

42. Nunes, M.R.; de Souza Maguerroski Castilho, M.; de Lima Veeck, A.P.; da Rosa, C.G.; Noronha, C.M.; Maciel, M.V.O.B.; Barreto, P.M. Antioxidant and antimicrobial methylcellulose films containingLippia alba extract and silver nanoparticles. Carbohydr. Polym. 2018, 192, 37-43. [CrossRef]

43. Huang, X.; Zhao, Z.; Fan, J.; Tan, Y.; Zheng, N. Amine-assisted synthesis of concave polyhedral platinum nanocrystals having $\{411\}$ high-index facets. J. Am. Chem. Soc. 2011, 133, 4718-4721. [CrossRef]

44. Jeyaraj, M.; Gurunathan, S.; Qasim, M.; Kang, M.H.; Kim, J.H. A Comprehensive Review on the Synthesis, Characterization, and Biomedical Application of Platinum Nanoparticles. Nanomaterials 2019, 9, 1719. [CrossRef]

45. Kim, J.; Takahashi, M.; Shimizu, T.; Shirasawa, T.; Kajita, M.; Kanayama, A.; Miyamoto, Y. Effects of a potent antioxidant, platinum nanoparticle, on the lifespan of Caenorhabditis elegans. Mech. Ageing Dev. 2008, 129, 322-331. [CrossRef]

46. Saitoh, Y.; Yoshimura, Y.; Nakano, K.; Miwa, N. Platinum nanocolloid-supplemented hydrogen dissolved water inhibits growth of human tongue carcinoma cells preferentially over normal cells. Exp. Oncol. 2009, 31, 156-162.

47. Tahir, K.; Ahmad, A.; Li, B.; Nazir, S.; Khan, A.U.; Nasir, T.; Khan, Z.U.H.; Naz, R.; Raza, M. Visible light photo catalytic inactivation of bacteria and photo degradation of methylene blue with $\mathrm{Ag} / \mathrm{TiO}_{2}$ nanocomposite prepared by a novel method. $J$. Photochem. Photobiol. B Biol. 2016, 162, 189-198. [CrossRef]

48. Miri, A.; Sarani, M. Biosynthesis, characterization and cytotoxic activity of $\mathrm{CeO}_{2}$ nanoparticles. Ceram. Int. 2018, 44, 12642-12647. [CrossRef]

49. Gunalan, S.; Sivaraj, R.; Rajendran, V. Green synthesized ZnO nanoparticles against bacterial and fungal pathogens. Prog. Nat. Sci. 2012, 22, 693-700. [CrossRef]

50. Stankic, S.; Suman, S.; Haque, F.; Vidic, J. Pure and multi metal oxide nanoparticles: Synthesis, antibacterial and cytotoxic properties. J. Nanobiotechnol. 2016, 14, 73. [CrossRef]

51. Hameed, A.S.H.; Karthikeyan, C.; Ahamed, A.P.; Thajuddin, N.; Alharbi, N.S.; Alharbi, S.A.; Ravi, G. In vitro antibacterial activity of $\mathrm{ZnO}$ and $\mathrm{Nd}$ doped $\mathrm{ZnO}$ nanoparticles against ESBL producing Escherichia coli and Klebsiella pneumoniae. Sci. Rep. 2016, 6, 24312. [CrossRef] 
52. Saratale, R.G.; Karuppusamy, I.; Saratale, G.D.; Pugazhendhi, A.; Kumar, G.; Park, Y.; Ghodake, G.S.; Bhargava, R.N.; Banu, J.R.; Shin, H.S. A comprehensive review on green nanomaterials using biological systems: Recent perception and their future applications. Colloids Surf. B Biointerfaces 2018, 170, 20-35. [CrossRef]

53. Pugazhendhi, T.N.; Edison, J.I.; Karuppusamy, I.; Kathirvel, B. Inorganic nanoparticles: A potential cancer therapy for human welfare. Int. J. Pharm. 2018, 539, 104-111. [CrossRef]

54. Taylor, J.; Rabe, T.; McGaw, L.; Jäger, A.; Van Staden, J. Towards the scientific validation of traditional medicinal plants. Plant Growth Regul. 2001, 34, 23-37. [CrossRef]

55. Mody, V.V.; Siwale, R.; Singh, A.; Mody, H.R. Introduction to metallic nanoparticles. J. Pharm. Bioall. Sci. 2010, 2, 282. [CrossRef] [PubMed]

56. Anand, A.S.; Prasad, D.N.; Singh, S.B.; Kohli, E. Chronic exposure of zinc oxide nanoparticles causes deviant phenotype in Drosophila melanogaster. J. Hazard. Mater. 2017, 327, 180-186. [CrossRef]

57. Sharma, V.; Anderson, D.; Dhawan, A. Zinc oxide nanoparticles induce oxidative DNA damage and ROS triggered mitochondria mediated apoptosis in human liver cells (HepG2). Apoptosis 2012, 17, 852-870. [CrossRef]

58. Rajakumar, G.; Iruvengadam, M.; Mydhili, G.; Gomathi, T.; Chung, I.M. Green approach for synthesis of zinc oxide nanoparticles from Andrographis paniculataleaf extract and evaluation of their antioxidant, anti-diabetic, and anti-inflammatory activities. Bioprocess Biosyst. Eng. 2018, 41, 21-30. [CrossRef]

59. Xiong, H.M. ZnO nanoparticles applied to bioimaging and drug delivery. Adv. Mater. 2013, 25, 5329-5335. [CrossRef]

60. Zhang, Y.; Nayak, T.R.; Hong, H.; Cai, W. Biomedical applications of zinc oxide nanomaterials. Curr. Mol. Med. 2013, 13, 1633-1645. [CrossRef]

61. Zhu, P.; Weng, Z.; Li, X.; Liu, X.; Wu, S.; Yeung, K.W.K.; Wang, X.; Cui, Z.; Yang, X.; Chu, P.K. Biomedical applications of functionalized $\mathrm{ZnO}$ nanomaterials: From biosensors to bioimaging. Adv. Mater. Interfaces 2016, 3, 1500494. [CrossRef]

62. Hameed, S.; Khalil, A.T.; Ali, M.; Numan, M.; Khamlich, S.; Shinwari, Z.K.; Maaza, M. Greener synthesis of ZnO and Ag-ZnO nanoparticles using Silybum marianum for diverse biomedical applications. Nanomedicine 2019, 14, 655-673. [CrossRef]

63. Moezzi, A.M.; McDonagh, M.B. Cortie Zinc oxide particles: Synthesis, properties and applications. Chem. Eng. J. 2012, 185, 1-22. [CrossRef]

64. Hatamie, A.; Khan, A.; Golabi, M.; Turner, A.P.; Beni, V.; Mak, W.C.; Sadollahkhani, Z.; Alnoor, H.; Zargar, B.; Bano, S.; et al. Zinc oxide nanostructure-modified textile and its application to biosensing, photocatalysis, and as antibacterial material. Langmuir 2015, 31, 10913-10921. [CrossRef]

65. Hu, X.; Cook, S.; Wang, P.; Hwang, H.M. In vitro evaluation of cytotoxicity of engineered metal oxide nanoparticles. Sci. Total Environ. 2009, 407, 3070-3072. [CrossRef]

66. Rasmussen, J.W.; Martinez, E.; Louka, P.; Wingett, D.G. Zinc oxide nanoparticles for selective destruction of tumor cells and potential for drug delivery applications. Expert Opin. Drug Deliv. 2010, 7, 1063-1077. [CrossRef]

67. Santhosh kumar, J.; Kumar, S.V.; Rajeshkumar, S. Synthesis of zinc oxide nanoparticles using plant leaf extract against urinary tract infection pathogen. Resour.-Effic. Technol. 2017, 3, 459-465.

68. Devi, H.S.; Singh, T.D. Synthesis of copper oxide nanoparticles by a novel method and its application in the degradation of methyl orange. Adv. Electr. Electron. Eng. 2014, 4, 83-88.

69. Bhattacharjee, A.; Ahmaruzzaman, M. CuO nanostructures: Facile synthesis and applications for enhanced photodegradation of organic compounds and reduction of pnitrophenol from aqueous phase. RSC Adv. 2016, 6, 41348-41363. [CrossRef]

70. Rakhshani, E. Preparation, characteristics and photovoltaic properties of cuprous oxide-A review. Solid-State Electron. 1986, 29, 7-17. [CrossRef]

71. Nabila, M.I.; Kannabiran, K. Biosynthesis, characterization and antibacterial activity of copper oxide nanoparticles (CuO NPs) from actinomycetes. Biocatal. Agric. Biotechnol. 2018, 15, 56-62. [CrossRef]

72. Mirza, A.U.; Khan, M.S.; Nami, S.A.; Kareem, A.; Rehman, S.; Bhat, S.A.; Nishat, N. Copper oxide nanomaterials Derived from Zanthoxylum armatum DC. and Berberis lyciumRoyle plant species: Characterization, assessment of free radical scavenging and antibacterial activity. Chem. Biodivers. 2019, 16, e1900145. [CrossRef]

73. Lee, S.W.; Woo, K.J.; Kim, C.S. Crystallographic and magnetic properties of iron oxide nanoparticles for applications in biomedicine. J. Magn. 2004, 9, 83-85. [CrossRef]

74. Muthukumar, H.; Chandrasekaran, N.I.; Mohammed, S.N.; Pichiah, S.; Manickam, M. Iron oxide nano-material: Physicochemical traits and in vitro antibacterial propensity against multidrug resistant bacteria. J. Ind. Eng. Chem. 2017, 45, 121-130. [CrossRef]

75. Chauhan, S.; Upadhyay, L.S.B. Biosynthesis of iron oxide nanoparticles using plant derivatives of Lawsoniainermis (Henna) and its surface modification for biomedical application. Nanotechnol. Environ. Eng. 2019, 4, 8. [CrossRef]

76. Soetaert, F.; Korangath, P.; Serantes, D.; Fiering, S.; Ivkov, R. Cancer therapy with iron oxide nanoparticles: Agents of thermal and immune therapies. Adv. Drug Deliv. Rev. 2020, 163, 65-83. [CrossRef]

77. Ahmad, W.; Kumar Jaiswal, K.; Amjad, M. Euphorbia herita leaf extract as a reducing agent in a facile green synthesis of iron oxide nanoparticles and antimicrobial activity evaluation. Inorg. Nano-Met. Chem. 2021, 51, 1147-1154. [CrossRef]

78. Yin, Z.F.; Wu, L.; Yang, H.G.; Su, Y.H. Recent progress in biomedical applications of titanium dioxide. Phys. Chem. Chem. Phys. 2013, 15, 4844-4858. [CrossRef] 
79. Wu, K.C.-W.; Yamauchi, Y.; Hong, C.-Y.; Yang, Y.-H.; Liang, Y.-H.; Funatsu, T.; Tsunoda, M. Biocompatible, surface functionalized mesoporous titania nanoparticles for intracellular imaging and anticancer drug delivery. Chem. Commun. 2011, 47, 5232-5234. [CrossRef]

80. Jayaseelan, C.; Rahuman, A.A.; Roopan, S.M.; Kirthi, A.V.; Venkatesan, J.; Kim, S.-K.; Iyappan, M.; Siva, C. Biological Approach to Synthesize TiO2 Nanoparticles Using Aeromonas hydrophila and Its Antibacterial Activity. Spectrochim. Acta Part A 2013, 107, 82-89. [CrossRef]

81. MuhdJulkapli, N.; Bagheri, S.; Bee Abd Hamid, S. Recent Advances in Heterogeneous Photocatalytic Decolorization of Synthetic Dyes. Sci. World J. 2014, 2014, 1-25. [CrossRef]

82. Visai, L.; De Nardo, L.; Punta, C.; Melone, L.; Cigada, A.; Imbriani, M.; Arciola, C.R. Titanium Oxide Antibacterial Surfaces in Biomedical Devices. Int. J. Artif. Organs 2011, 34, 929-946. [CrossRef]

83. Ren, W.; Zeng, L.; Shen, Z.; Xiang, L.; Gong, A.; Zhang, J.; Mao, C.; Li, A.; Paunesku, T.; Woloschak, G.E.; et al. Enhanced doxorubicin transport to multidrug resistant breast cancer cells via $\mathrm{TiO}_{2}$ nanocarriers. RSC Adv. 2013, 3, 20855-20861. [CrossRef]

84. Velayutham, K.; Rahuman, A.A.; Rajakumar, G.; Santhoshkumar, T.; Marimuthu, S.; Jayaseelan, C.; Bagavan, A.; Kirthi, A.V.; Kamaraj, C.; Zahir, A.A. Evaluation of Catharanthus Roseus Leaf Extract-mediated Biosynthesis of Titanium Dioxide Nanoparticles Against Hippobosca Maculata and Bovicola Ovis. Parasitol. Res. 2012, 111, 2329-2337. [CrossRef]

85. Marimuthu, S.; Rahuman, A.A.; Jayaseelan, C.; Kirthi, A.V.; Santhoshkumar, T.; Velayutham, K.; Bagavan, A.; Kamaraj, C.; Elango, G.; Iyappan, M. Acaricidal Activity of Synthesized Titanium Dioxide Nanoparticles Using Calotropis Gigantea Against Rhipicephalus microplus and Haemaphysalisbispinosa. Asian Pacific J. Trop. Med. 2013, 6, 682-688. [CrossRef]

86. Rajakumar, G.; Rahuman, A.A.; Priyamvada, B.; Khanna, V.G.; Kumar, D.K.; Sujin, P. EcliptaProstrata Leaf Aqueous Extract Mediated Synthesis of Titanium Dioxide Nanoparticles. Mater. Lett. 2012, 68, 115-117. [CrossRef]

87. Durairaj, B.; Xavier, T.; Muthu, S. Fungal Generated Titanium Dioxide Nanoparticles: A Potent Mosquito (Aedesaegypti) Larvicidal Agent. Sch. Acad. J. Biosci. 2014, 2, 651-658.

88. Srinivasan, M.; Venkatesan, M.; Arumugam, V.; Natesan, G.; Saravanan, N.; Murugesan, S.; Pugazhendhi, A. Green synthesis and characterization of titanium dioxide nanoparticles (TiO2 NPs) using Sesbania grandiflora and evaluation of toxicity in zebrafish embryos. Process Biochem. 2019, 80, 197-202. [CrossRef]

89. El-Shabouri, M.H. Positively charged nanoparticles for improving the oral bioavailability of cyclosporin-A. Int. J. Pharm. 2002, 249, 101-108. [CrossRef]

90. Hu, L.; Tang, X.; Cui, F. Solid lipid nanoparticles (SLNs) to improve oral bioavailability of poorly soluble drugs. J. Pharm. Pharmacol. 2004, 56, 1527-1535. [CrossRef]

91. Ju, S.; Mu, J.; Dokland, T.; Zhuang, X.; Wang, Q.; Jiang, H.; Xiang, X.; Deng, Z.B.; Wang, B.; Zhang, L.; et al. Grape exosome-like nanoparticles induce intestinal stem cells and protect mice from DSS-induced colitis. Mol. Ther. 2013, 21, 1345-1357. [CrossRef]

92. Król, A.; Pomastowski, P.; Rafińska, K.; Railean-Plugaru, V.; Buszewski, B. Zinc oxide nanoparticles: Synthesis, antiseptic activity and toxicity mechanism. Adv. Colloid Interf. Sci. 2017, 249, 37-52. [CrossRef]

93. Hano, C.; Abbasi, B.H. Plant-Based Green Synthesis of Nanoparticles: Production, Characterization and Applications. Biomolecules 2022, 12, 31. [CrossRef]

94. Shivashankar, M.; Sisodia, G. Biosynthesis of silver nanoparticles obtained from plant extracts of Moringa oleifera. Int. J. Life Sci. Biotechnol. Pharm. Res. 2012, 1, 182-185.

95. Paul, S.; Basak, P.; Majumder, R.; Mukherjee, A.; Ghosh, J.; Patra, S.; Jana, N.K. Biochemical estimation of Moringaoleifera leaf extract for synthesis of silver nanoparticle mediated drug delivery system. J. Plant Biochem. Biotechnol. 2020, 29, 86-93. [CrossRef]

96. Sharma, A.K.; Swami, A.K.; Jangir, D.; Saran, M.; Upadhyay, T.K.; Prajapat, R.K.; Mathur, M. An eco-friendly green synthesis of tungsten nanoparticles from Moringa oleifera Lam. and their pharmacological studies. GMJ 2020, 31, 719-725.

97. Sivaranjani, V.; Philominathan, P.J.W.M. Synthesize of Titanium dioxide nanoparticles using Moringa oleifera leaves and evaluation of wound healing activity. Wound Med. 2016, 12, 1-5. [CrossRef]

98. Sasidharan, S.; Pottail, L. Antimicrobial activity of metal and non-metallic nanoparticles from Cyperus rotundus root extract on infectious disease causing pathogens. J. Plant Biochem. Biotechnol. 2020, 29, 134-143. [CrossRef]

99. Yasmin, A.; Ramesh, K.; Rajeshkumar, S. Optimization and stabilization of gold nanoparticles by using herbal plant extract with microwave heating. Nano Converg. 2014, 1, 12. [CrossRef]

100. Adavallan, K.; Krishnakumar, N. Mulberry leaf extract mediated synthesis of gold nanoparticles and its anti-bacterial activity against human pathogens. Adv. Nat. Sci. Nanosci. Nanotechnol. 2014, 5, 025018. [CrossRef]

101. Shankar, S.S.; Ahmed, A.; Akkamwar, B.; Sastry, M.; Rai, A.; Singh, A. Biological synthesis of triangular gold nanoprism. Nature 2004, 3, 482. [CrossRef]

102. Ponarulselvam, S.; Panneerselvam, C.; Murugan, K.; Aarthi, N.; Kalimuthu, K.; Thangamani, S. Synthesis of silver nanoparticles using leaves of Catharanthus roseus Linn. G. Don and their antiplasmodial activities. Asian Pac. J. Trop. Biomed. 2012, 2, 574-580. [CrossRef]

103. Suresh, U.; Murugan, K.; Benelli, G.; Nicoletti, M.; Barnard, D.R.; Panneerselvam, C.; Chandramohan, B. Tackling the growing threat of dengue: Phyllanthus niruri-mediated synthesis of silver nanoparticles and their mosquitocidal properties against the dengue vector Aedesaegypti (Diptera: Culicidae). Parasitol. Res. 2015, 114, 1551-1562. [CrossRef] [PubMed]

104. Rane, R.V.; Meenakshi, K.; Shah, M.; Georg, I.A. Biological synthesis of silver nanoparticles using Abelmoschusmoschatus. Indian J. Biotechnol. 2014, 13, 342-346. 
105. Wen, X.; Wang, Q.; Dai, T.; Shao, J.; Wu, X.; Jiang, Z.; Jiang, C. Identification of possible reductants in the aqueous leaf extract of mangrove plant Rhizophora apiculata for the fabrication and cytotoxicity of silver nanoparticles against human osteosarcoma MG-63 cells. Mater. Sci. Eng. C 2020, 116, 111252. [CrossRef] [PubMed]

106. Rajput, S.; Kumar, D.; Agrawal, V. Green synthesis of silver nanoparticles using Indian Belladonna extract and their potential antioxidant, anti-inflammatory, anticancer and larvicidal activities. Plant Cell Rep. 2020, 39, 921-939. [CrossRef]

107. Tippayawat, P.; Phromviyo, N.; Boueroy, P.; Chompoosor, A. Green synthesis of silver nanoparticles in aloe vera plant extract prepared by a hydrothermal method and their synergistic antibacterial activity. Peer J. 2016, 4, e2589. [CrossRef]

108. Vélez, E.; Campillo, G.; Morales, G.; Hincapié, C.; Osorio, J.; Arnache, O. Silver nanoparticles obtained by aqueous or ethanolic aloe vera extracts: An assessment of the antibacterial activity and mercury removal capability. J. Nanomater. 2018, 2018, 7215210. [CrossRef]

109. Ramteke, C.; Chakrabarti, T.; Sarangi, B.K.; Pandey, R.A. Synthesis of silver nanoparticles from the aqueous extract of leaves of Ocimum sanctum for enhanced antibacterial activity. J. Chem. 2013, 2013, 278925. [CrossRef]

110. Nancy, B.A.; Elumalai, K. Synthesis of Silver Nanoparticles Using Pelargonium graveolens Essential Oil and Anti-Fungal Activity. Int. J. Pharm. Biol. Sci.-IJPBSTM 2019, 9, 176-185.

111. Bere, A.W.; Mulati, O.; Kimotho, J.; Ng'ong'a, F. Carica papaya Leaf Extract Silver Synthesized Nanoparticles Inhibit Dengue Type 2 Viral Replication In Vitro. Pharmaceuticals 2021, 14, 718. [CrossRef]

112. Gunti, L.; Dass, R.S.; Kalagatur, N.K. Phytofabrication of selenium nanoparticles from Emblica officinalis fruit extract and exploring its biopotential applications: Antioxidant, antimicrobial, and biocompatibility. Front. Microbiol. 2019, 10, 931. [CrossRef]

113. Goyal, P.; Bhardwaj, A.; Mehta, B.K.; Mehta, D. Research article green synthesis of zirconium oxide nanoparticles $\left(\mathrm{ZrO}_{2} \mathrm{NPs}\right)$ using Helianthus annuus seed and their antimicrobial effects. J. Indian Chem. Soc. 2021, 98, 100089. [CrossRef]

114. Muhammad, W.; Khan, M.A.; Nazir, M.; Siddiquah, A.; Mushtaq, S.; Hashmi, S.S.; Abbasi, B.H. Papaver somniferum L. mediated novel bioinspired lead oxide $(\mathrm{PbO})$ and iron oxide $\left(\mathrm{Fe}_{2} \mathrm{O}_{3}\right)$ nanoparticles: In-vitro biological applications, biocompatibility and their potential towards HepG2 cell line. Mater. Sci. Eng. C 2019, 103, 109740. [CrossRef]

115. Mani, M.; Pavithra, S.; Mohanraj, K.; Kumaresan, S.; Alotaibi, S.S.; Eraqi, M.M.; Kaviyarasu, K. Studies on the spectrometric analysis of metallic silver nanoparticles (Ag NPs) using Basella alba leaf for the antibacterial activities. Environ. Res. 2021, 199, 111274. [CrossRef]

116. Yu, Z.; Liu, J.; He, H.; Wang, Y.; Zhao, Y.; Lu, Q.; Peng, Y. Green synthesis of silver nanoparticles with black rice (Oryza sativa L.) extract endowing carboxymethyl chitosan modified cotton with high anti-microbial and durable properties. Cellulose 2021, 28, 1827-1842. [CrossRef]

117. Chauhan, N.; Tyagi, A.K.; Kumar, P.; Malik, A. Antibacterial potential of Jatropha curcas synthesized silver nanoparticles against food borne pathogens. Front. Microbiol. 2016, 7, 1748. [CrossRef]

118. Vimalraj, S.; Ashokkumar, T.; Saravanan, S. Biogenic gold nanoparticles synthesis mediated by Mangifera indica seed aqueous extracts exhibits antibacterial, anticancer and anti-angiogenic properties. Biomed. Pharmacother. 2018, 440-448. [CrossRef]

119. Qamar, H.; Rehman, S.; Chauhan, D.K.; Tiwari, A.K.; Upmanyu, V. Green synthesis, characterization and antimicrobial activity of copper oxide nanomaterial derived from Momordica charantia. Int. J. Nanomed. 2020, 15, 2541. [CrossRef]

120. Hua, S.; de Matos, M.B.C.; Metselaar, J.M.; Storm, G. Current trends and challenges in the clinical translation of nanoparticulate nanomedicines: Pathways for translational development and commercialization. Front. Pharmacol. 2018, 9, 790. [CrossRef]

121. Bhat, R.; Sharanabasava, V.G.; Deshpande, R.; Shetti, U.; Sanjeev, G.; Venkataraman, A. Photo-bio-synthesis of irregular shaped functionalized gold nanoparticles using edible mushroom Pleurotusflorida and its anticancer evaluation. J. Photochem. 2013, 125, 63-69.

122. Guo, Y.; Sun, L.; Chen, X.P.; Zhang, D.S. Oxidative stress, mitochondrial damage and neurodegenerative diseases. Neural. Regen. Res. 2013, 8, 2003-2014.

123. Arakha, M.; Roy, J.; Nayak, P.S.; Mallick, B.; Jha, S. Zinc oxide nanoparticle energy band gap reduction triggers the oxidative stress resulting into autophagy-mediated apoptotic cell death. Free. Radic. Biol. Med. 2017, 110, 42-53. [CrossRef]

124. Asharani, P.V.; Hande, M.P.; Valiyaveettil, S. Anti-proliferative activity of silver nanoparticles. BMC Cell Biol. $2009,10,65$. [CrossRef]

125. Raja, G.; Kim, S.; Yoon, D.; Yoon, C.; Kim, S. ${ }^{1} \mathrm{H}$ NMR based metabolomics studies of the toxicity of titanium dioxide nanoparticles in zebrafish (Danio rerio). Bull. Korean Chem. Soc. 2018, 39, 33-39. [CrossRef]

126. Saeed, M.; Iqbal, M.Z.; Ren, W.Z.; Xia, Y.Z.; Liu, C.; Khan, W.S.; Wu, A.G. Controllable synthesis of $\mathrm{Fe}_{3} \mathrm{O}_{4}$ nanoflowers: Enhanced imaging guided cancer therapy and comparison of photothermal efficiency with black-TiO 2 . J. Mater. Chem. B. 2018, 6, 3800-3810. [CrossRef]

127. Iqbal, M.Z.; Ren, W.Z.; Saeed, M.; Chen, T.X.; Ma, X.H.; Yu, X.; Zhang, J.C.; Zhang, L.L.; Li, A.G.; Wu, A.G. A facile fabrication route for binary transition metal oxide-based Janus nanoparticles for cancer theranostic applications. Nano Res. 2018, 11, 5735-5750. [CrossRef]

128. Sun, Q.; Zhou, Z.; Qiu, N.; Shen, Y. Rational design of cancer nanomedicine: Nanoproperty integration and synchronization. Adv. Mater. 2017, 29, 1606628. [CrossRef]

129. Hoffman, A.J.; Yee, H.; Mills, G.; Hoffmann, M.R. Photoinitiated polymerization of methyl methacrylate using Q-sized zinc oxide colloids. J. Phys. Chem. 1992, 96, 5540-5546. [CrossRef]

130. Schmid, G. Large clusters and colloids. Metals in the embryonic state. Chem. Rev. 1992, 92, 1709-1727. [CrossRef] 
131. Scalbert, A.; Manach, C.; Morand, C.; Rémésy, C.; Jiménez, L. Dietary polyphenols and the prevention of diseases. Crit. Rev. Food Sci. Nutr. 2005, 45, 287-306. [CrossRef]

132. Rah, D.K.; Han, D.W.; Baek, H.S.; Hyon, S.H.; Park, B.Y.; Park, J.C. Protection of rabbit kidney from ischemia/reperfusion injury by green tea polyphenol pretreatment. Arch. Pharm. Res. 2007, 30, 1447-1454. [CrossRef]

133. Mohammadinejad, R.; Karimi, S.; Iravani, S.; Varma, R.S. Plant-derived nanostructures: Types and applications. Green Chem. 2016, 18, 20-52. [CrossRef]

134. Lin, N.; Dufresne, A. Nanocellulose in biomedicine: Current status and future prospect. Eur. Polym. J. 2014, 59, 302-325. [CrossRef]

135. Karimi, S.; Tahir, P.M.; Karimi, A.; Dufresne, A.; Abdulkhani, A. Kenaf bast cellulosic fibers hierarchy: A comprehensive approach from micro to nano. Carbohydr. Polym. 2014, 101, 878-885. [CrossRef] [PubMed]

136. Berglund, L. Cellulose-based nanocomposites. In Natural Fibers, Biopolymers and Biocomposites; Mohanty, A.K., Misra, M., Drzal, L.T., Eds.; CRC Press: Boca Raton, FL, USA, 2005.

137. Jha, A.K.; Prasad, K.; Prasad, K.; Kulkarni, A.R. Plant system: Nature's nanofactory. Colloids Surf. B Biointerfaces 2009, 73, $219-223$. [CrossRef]

138. Kesharwani, J.; Yoon, K.Y.; Hwang, J.; Rai, M. Phytofabrication of silver nanoparticles by leaf extract of Datura metel: Hypothetical mechanism involved in synthesis. J. Bionanosci. 2009, 3, 39-44. [CrossRef]

139. Xu, H.; Jiang, Q.; Reddy, N.; Yang, Y. Hollow nanoparticles from zein for potential medical applications. J. Mater. Chem. 2011, 21, 18227-18235. [CrossRef]

140. Muramatsu, H.; Kim, Y.A.; Yang, K.S.; Cruz-Silva, R.; Toda, I.; Yamada, T.; Saitoh, H. Rice husk-derived graphene with nano-sized domains and clean edges. Small 2014, 10, 2766-2770. [CrossRef]

141. Chen, X.W.; Timpe, O.; Hamid, S.B.; Schlögl, R.; Su, D.S. Direct synthesis of carbon nanofibers on modified biomass-derived activated carbon. Carbon 2009, 47,340-343. [CrossRef]

142. Zhu, J.; Jia, J.; Kwong, F.L.; Ng, D.H.L.; Tjong, S.C. Synthesis of multiwalled carbon nanotubes from bamboo charcoal and the roles of minerals on their growth. Biomass Bioenergy 2012, 36, 12-19. [CrossRef]

143. Xia, L.; Lenaghan, S.C.; Wills, A.B.; Chen, Y.; Zhang, M. Evaluation of the nanofibrillar structure of Dioscorea opposite extract for cell attachment. Colloids Surf. B Biointerfaces 2011, 88, 425-431. [CrossRef]

144. Wang, B.; Zhuang, X.; Deng, Z.B.; Jiang, H.; Mu, J.; Wang, Q.; Xiang, X.; Guo, H.; Zhang, L.; Dryden, G. Targeted drug delivery to intestinal macrophages by bioactive nanovesicles released from grapefruit. Mol. Ther. 2014, 22, 522-534. [CrossRef]

145. Voirin, C.; Caillol, S.; Sadavarte, N.V.; Tawade, B.V.; Boutevin, B.; Wadgaonkar, P.P. Functionalization of cardanol: Towards biobased polymers and additives. Polym. Chem. 2014, 5, 3142-3162. [CrossRef]

146. Balachandran, V.S.; Jadhav, S.R.; Vemula, P.K.; John, G. Recent advances in cardanol chemistry in a nutshell: From a nut to nanomaterials. Chem. Soc. Rev. 2013, 42, 427-438. [CrossRef]

147. Liu, N.; Huo, K.; McDowell, M.T.; Zhao, J.; Cui, Y. Rice husks as a sustainable source of nanostructured silicon for high performance Li-ion battery anodes. Sci. Rep. 2013, 3, 1919. [CrossRef]

148. Kim, J.E.; Lee, J.; Jang, M.; Kwak, M.H.; Go, J.; Kho, E.K.; Song, S.H.; Sung, J.E.; Lee, J.; Hwang, D.Y. Accelerated healing of cutaneous wounds using phytochemically stabilized gold nanoparticle deposited hydrocolloid membranes. Biomater. Sci. 2015, 3 , 509-519. [CrossRef]

149. Naraginti, S.; Kumari, P.L.; Das, R.K.; Sivakumar, A.; Patil, S.H.; Andhalkar, V.V. Amelioration of excision wounds by topical application of green synthesized, formulated silver and gold nanoparticles in albino Wistar rats. Mater. Sci. Eng. C Mater. Biol. Appl. 2016, 62, 293-300. [CrossRef]

150. Krychowiak, M.; Grinholc, M.; Banasiuk, R.; Krauze-Baranowska, M.; Głód, D.; Kawiak, A.; Królicka, A. Combination of silver nanoparticles and Droserabinata extract as a possible alternative for antibiotic treatment of burn wound infections caused by resistant Staphylococcus aureus. PLoS ONE 2014, 9, e115727.

151. Ramazan, E. Advances in fabric structures for wound care. In Advanced Textiles for Wound Care; Rajendran, S., Ed.; Woodhead Publishing: Cambridge, UK, 2019; pp. 509-540.

152. Aderibigbe, B.A.; Buyana, B. Alginate in Wound Dressings. Pharmaceutics 2018, 10, 42. Available online: https://www.ncbi.nlm. nih.gov/pmc/articles/PMC6027439/ (accessed on 4 January 2022). [CrossRef]

153. Tayi, A.S.; Pashuck, E.T.; Newcomb, C.J.; McClendon, M.T.; Stupp, S.I. Electrospinning bioactive supramolecular polymers from water. Biomacromolecules 2014, 15, 1323-1327. [CrossRef]

154. Dias Antunes, M.; da Silva Dannenberg, G.; Fiorentini, A.M.; Pinto, V.Z.; Lim, L.T.; daRosaZavareze, E.; Dias, A.R.G. Antimicrobial electrospun ultrafine fibers from zein containing eucalyptus essential oil/cyclodextrin inclusion complex. Int. J. Biol. Macromol. 2017, 104, 874-882. [CrossRef]

155. Moreno, M.A.; Orqueda, M.E.; Go'mez-Mascaraque, L.G.; Isla, M.I.; Lo'pez-Rubio, A. Crosslinked electrospun zein-based food packaging coatings containing bioactive chilto fruit extracts. Food Hydrocoll. 2019, 95, 496-505. [CrossRef]

156. Miguel, S.P.; Sequeira, R.S.; Moreira, A.F.; Cabral, C.S.; Mendonça, A.G.; Ferreira, P.; Correia, I.J. An overview of electrospun membranes loaded with bioactive molecules for improving the wound healing process. Eur. J. Pharm. Biopharm. 2019, 139, 1-22 [CrossRef] [PubMed]

157. Suwantong, O.; Opanasopit, P.; Ruktanonchai, U.; Supaphol, P. Electrospun cellulose acetate fiber mats containing curcumin and release characteristic of the herbal substance. Polymer 2007, 48, 7546-7557. [CrossRef] 
158. Momtazi-Borojeni, A.A.; Haftcheshmeh, S.M.; Esmaeili, S.A.; Johnston, T.P.; Abdollahi, E.; Sahebkar, A. Curcumin: A natural modulator of immune cells in systemic lupus erythematosus. Autoimmun. Rev. 2017, 17, 125-135. [CrossRef]

159. Marsell, R.; Einhorn, T.A. The biology of fracture healing. Injury 2011, 42, 551-555. [CrossRef]

160. Nicolin, V.; De Tommasi, N.; Nori, S.L.; Costantinides, F.; Berton, F.; Di Lenarda, R. Modulatory effects of plant polyphenols on bone remodeling: A prospective view from the bench to bedside. Front. Endocrinol. 2019, 10, 494. [CrossRef]

161. Park, Y.H.; Han, D.W.; Suh, H.; Ryu, G.H.; Hyon, S.H.; Cho, B.K.; Park, J.C. Protective effects of green tea polyphenol against reactive oxygen species-induced oxidative stress in cultured rat calvarial osteoblast. Cell Biol. Toxicol. 2003, 19, 325-337. [CrossRef]

162. Vu, M.N.; Kelly, H.G.; Kent, S.J.; Wheatley, A.K. Current and future nanoparticle vaccines for COVID-19. EBioMedicine 2021, 74, 103699. [CrossRef]

163. Jagessar, R.C. Plant Extracts Based Nanoparticles, Potential Nanomedicine in Fight against COVID-19. J. Nanosci. Res. Rep. 2020, $2,1-4$.

164. Medicago and GSK Announce Positive Phase 3 Efficacy and Safety Results for Adjuvanted Plant-Based COVID-19 Vaccine Candidate. Available online: https:/ / www.gsk.com/en-gb/media/press-releases/medicago-and-gsk-announce-positive-phase3-efficacy-and-safety-results / (accessed on 4 January 2022).

165. Prabha, S.; Durgalakshmi, D.; Rajendran, S.; Lichtfouse, E. Plant-derived silica nanoparticles and composites for biosensors, bioimaging, drug delivery and supercapacitors: A review. Environ. Chem. Lett. 2020, 19, 1667-1691. [CrossRef]

166. Parveen, K.; Banse, V.; Ledwani, L. Green synthesis of nanoparticles: Their advantages and disadvantages. In Proceedings of the 2nd International Conference on Emerging Technologies: Micro to Nano 2015, Rajasthan, India, 24-25 October 2015; Volume 1724, p. 020048. 